\title{
Polynomial ring representations of endomorphisms of exterior powers
}

\author{
Ommolbanin Behzad ${ }^{1} \cdot$ André Contiero $^{2} \cdot$ Letterio Gatto $^{3}\left(\mathbb{D} \cdot\right.$ Renato Vidal Martins $^{2}$
}

Received: 11 August 2020 / Accepted: 28 November 2020 / Published online: 3 February 2021

(c) The Author(s) 2021

\begin{abstract}
An explicit description of the ring of the rational polynomials in $r$ indeterminates as a representation of the Lie algebra of the endomorphisms of the $k$-th exterior power of a countably infinite-dimensional vector space is given. Our description is based on results by Laksov and Throup concerning the symmetric structure of the exterior power of a polynomial ring. Our results are based on approximate versions of the vertex operators occurring in the celebrated bosonic vertex representation, due to Date, Jimbo, Kashiwara and Miwa, of the Lie algebra of all matrices of infinite size, whose entries are all zero but finitely many.
\end{abstract}

Keywords Hasse-Schmidt derivations and vertex operators on exterior algebras $\cdot$ Bosonic and fermionic representations by Date-Jimbo-Kashiwara-Miwa $\cdot$ Symmetric functions

Mathematics Subject Classification $14 \mathrm{M} 15 \cdot 15 \mathrm{~A} 75 \cdot 05 \mathrm{E} 05 \cdot 17 \mathrm{~B} 69$

Work sponsored by Finanziamento Diffuso della Ricerca, No. 53_RBA17GATLET del Politecnico di Torino; Progetto di Eccellenza Dipartimento di Scienze Matematiche, 2018-2022 No. E11G18000350001, INDAM-GNSAGA e PRIN "Geometria delle Varietà Algebriche".

Letterio Gatto

letterio.gatto@polito.it

Ommolbanin Behzad

behzad@iasbs.ac.ir

André Contiero

contiero@ufmg.br

Renato Vidal Martins

vidalmartins@ufmg.br

1 Institute for Advanced Studies in Basic Sciences, Zanjan, Iran

2 Universidade Federal de Minas Gerais, Belo Horizonte, MG, Brazil

3 Dipartimento di Scienze Matematiche, Politecnico di Torino, Turin, Italy 


\section{Introduction}

\subsection{Statement of the main result}

The purpose of this paper is to supply an explicit description of the polynomial ring $B_{r}:=\mathbb{Q}\left[e_{1}, \ldots, e_{r}\right]$ as a module over the Lie algebra of endomorphisms of $k$-th exterior powers of a vector space $V:=\bigoplus_{i \geq 0} \mathbb{Q} \cdot b_{i}$ of infinite countable dimension.

Let $\beta_{j}: V \rightarrow \mathbb{Q}$ be the unique linear form such that $\beta_{j}\left(b_{i}\right)=\delta_{j i}$ so that $V^{*}:=\bigoplus_{j \geq 0} \mathbb{Q} \cdot \beta_{j}$ is the restricted dual of $V$. Write the $r$-th exterior powers of $V$ and $V^{*}$, respectively, as $\bigwedge^{r} V:=\bigoplus_{\lambda \in \mathcal{P}_{r}} \mathbb{Q} \cdot[\mathbf{b}]_{\lambda}^{r}$ and $\bigwedge^{r} V^{*}:=\bigoplus_{\lambda \in \mathcal{P}_{r}} \mathbb{Q} \cdot[\boldsymbol{\beta}]_{\lambda}^{r}$, with $[\mathbf{b}]_{\lambda}^{r}:=b_{r-1+\lambda_{1}} \wedge \cdots \wedge b_{\lambda_{r}}$ and $[\boldsymbol{\beta}]_{\lambda}^{r}\left([\mathbf{b}]_{\mu}^{r}\right)=\delta_{\lambda, \mu}$, where $\lambda$ and $\boldsymbol{\mu}$ range over the set $\mathcal{P}_{r}$ of all the partitions of length at most $r$.

A strict relative of $\bigwedge^{r} V$ is the vector space $B_{r}$, which can be identified with the ring of symmetric polynomials in $r$ indeterminates. It is well known that it possesses a $\mathbb{Q}$ -basis formed by certain Schur determinants $S_{\lambda}:=S_{\lambda}\left(e_{1}, \ldots, e_{r}\right)$ (Cf. Sect. 2.4, adopting the notation of $\left[12\right.$, p. 41]). The $\mathbb{Q}$-linear extension of the set map $S_{\lambda} \mapsto[\mathbf{b}]_{\lambda}^{r}$ yields a $\mathbb{Q}$-vector space isomorphism $B_{r} \rightarrow \bigwedge^{r} V$, sending $1 \mapsto[\mathbf{b}]_{0}^{r}:=b_{r-1} \wedge \cdots \wedge b_{0}$. It is convenient to phrase it by saying that $\bigwedge^{r} V$ is a free $B_{r}$-module of rank 1 generated by $[\mathbf{b}]_{0}^{r}$, such that $[\mathbf{b}]_{\lambda}^{r}=\Delta_{\lambda}\left(H_{r}\right)[\mathbf{b}]_{0}^{r}$ (Sect. 2.3).

Let $\operatorname{gl}\left(\bigwedge^{k^{\lambda}} V\right)$ be the Lie algebra of the endomorphisms of $\bigwedge^{k} V$ vanishing at $[\mathbf{b}]_{\lambda}^{k}$ for all but finitely many partitions $\lambda \in \mathcal{P}_{k}$ and denote by $\mathcal{E}_{\mu, v}^{k}$ the elementary endomorphism $[\mathbf{b}]_{\mu}^{k} \otimes[\boldsymbol{\beta}]_{\nu}^{k}$, so that:

$$
g l\left(\bigwedge^{k} V\right)=\bigwedge^{k} V \otimes \bigwedge^{k} V^{*}=\bigoplus_{\mu, \nu \in \mathcal{P}_{k}} \mathbb{Q} \cdot \mathcal{E}_{\mu, \nu}^{k} .
$$

For all $k, r \geq 0$, we consider the $B_{r}$-representation of $g l\left(\bigwedge^{k} V\right)$, which we understand as the action:

$$
\left.\left(\mathcal{E}_{\boldsymbol{\mu}, \boldsymbol{\nu}}^{k} \Delta_{\lambda}\left(H_{r}\right)\right)[\mathbf{b}]_{0}^{r}=[\mathbf{b}]_{\mu}^{k} \wedge\left([\boldsymbol{\beta}]_{\nu}^{k}\right\lrcorner[\mathbf{b}]_{\lambda}^{r}\right),
$$

where $\left.[\boldsymbol{\beta}]_{v}^{k}\right\lrcorner: \bigwedge^{r} V \rightarrow \bigwedge^{r-k} V$ is the standard contraction operator (Sect. 2.2).

To express the $g l\left(\bigwedge^{k} V\right)$-action (1) on $B_{r}$ through a compact formula, a standard philosophy suggests to use generating functions.

To this purpose, let us introduce some notation. Let $\mathbf{z}_{k}:=\left(z_{1}, \ldots, z_{k}\right)$ and $\mathbf{w}_{k}:=\left(w_{1}, \ldots, w_{k}\right)$ be two sets of formal variables. The $k$-tuples of the formal inverses $\left(z_{1}^{-1}, \ldots, z_{k}^{-1}\right)$ and $\left(w_{1}^{-1}, \ldots, w_{k}^{-1}\right)$ will be denoted by $\mathbf{z}_{k}^{-1}$ and $\mathbf{w}_{k}^{-1}$ respectively. If $\mathbf{u}:=\left(u_{1}, \ldots, u_{k}\right)$ are arbitrary formal variables, denote by $p_{i}\left(\mathbf{u}_{k}\right)$ the power sum $u_{1}^{i}+\cdots+u_{k}^{i}$ of degree $i$. The standard notation $s_{\boldsymbol{\mu}}\left(\mathbf{z}_{k}\right)$ and $s_{\boldsymbol{\nu}}\left(\mathbf{w}_{k}^{-1}\right)$ stands for the symmetric Schur polynomials in the $\mathbf{z}_{k}$ and $\mathbf{w}_{k}^{-1}$ (See [12, p. 40]). Let $E_{r}(z):=1-e_{1} z+\cdots+(-1)^{r} e_{r} z^{r} \in B_{r}[z]$, set by convention $b_{j}=0$ if $j<0$ and denote by $\sigma_{-1}$ the locally nilpotent endomorphism of $V$ mapping $b_{j} \mapsto b_{j-1}$ for all $j \geq 0$. Let $\delta: \operatorname{End}(V) \mapsto \operatorname{End}(\bigwedge V)$ be the natural representation of $\operatorname{End}(V)$ as a Lie algebra of (even) derivations of $\bigwedge V$.

The main ingredients to state our main result are certain vertex operators $\Gamma\left(\mathbf{z}_{k}\right), \Gamma^{*}\left(\mathbf{z}_{k}\right): \bigwedge V \rightarrow \bigwedge V\left[\mathbf{z}_{k}, \mathbf{z}_{k}^{-1}\right]$ acting on the exterior algebra $\bigwedge V$. They are introduced in Definition 4.3 as products of Schubert derivations, and studied in more detail only in Sects. 6 and 7. However, if $r$ is big with respect to the length of the partition $\lambda$ they can be explicitly written as 


$$
\Gamma\left(\mathbf{z}_{k}\right)[\mathbf{b}]_{\lambda}^{r}:=\prod_{j=1}^{k} \frac{1}{E_{r}\left(z_{j}\right)} \exp \left(-\sum_{i \geq 1} \frac{1}{i} \delta\left(\sigma_{-1}^{i}\right) p_{i}\left(\mathbf{z}_{k}^{-1}\right)\right)[\mathbf{b}]_{\lambda}^{r+k}
$$

and

$$
\Gamma^{*}\left(\mathbf{w}_{k}\right)[\mathbf{b}]_{\lambda}^{r}:=\prod_{j=1}^{k} E_{r}\left(w_{j}\right) \exp \left(\sum_{i \geq 1} \frac{1}{i} \delta\left(\sigma_{-1}^{i}\right) p_{i}\left(\mathbf{w}_{k}^{-1}\right)\right)[\mathbf{b}]_{\lambda}^{r-k} .
$$

Consider now the generating formal power series

$$
\mathcal{E}\left(\mathbf{z}_{k}, \mathbf{w}_{k}^{-1}\right)=\sum_{\mu, \nu \in \mathcal{P}_{k}} \mathcal{E}_{\mu \nu}^{k} \cdot s_{\mu}\left(\mathbf{z}_{k}\right) s_{\nu}\left(\mathbf{w}_{k}^{-1}\right): B_{r} \rightarrow B_{r}\left[\mathbf{z}_{k}, \mathbf{w}_{k}^{-1}\right]
$$

defined by the equality:

$$
\left.\left(\mathcal{E}\left(\mathbf{z}_{k}, \mathbf{w}_{k}^{-1}\right) S_{\lambda}\right)[\mathbf{b}]_{0}^{r}=\sum_{\mu . v \in \mathcal{P}_{k}} s_{\mu}\left(\mathbf{z}_{k}\right) s_{\nu}\left(\mathbf{w}_{k}^{-1}\right)[\mathbf{b}]_{\lambda}^{k} \wedge\left([\boldsymbol{\beta}]_{\nu}^{k}\right\lrcorner[\mathbf{b}]_{\lambda}^{r}\right) .
$$

Main Theorem. For all $k, r \geq 0$ and all $\lambda \in \mathcal{P}_{r}$, the action of $\mathcal{E}\left(\mathbf{z}_{k}, \mathbf{w}_{k}^{-1}\right)$ on the basis element $S_{\lambda}$ of $B_{r}$ is given by:

$$
\left(\mathcal{E}\left(\mathbf{z}_{k}, \mathbf{w}_{k}^{-1}\right) S_{\lambda}\right)[\mathbf{b}]_{0}^{r}=\prod_{j=1}^{k}\left(\frac{z_{j}}{w_{j}}\right)^{r-k} \cdot \Gamma\left(\mathbf{z}_{k}\right) \Gamma^{*}\left(\mathbf{w}_{k}\right)[\mathbf{b}]_{\lambda}^{r} .
$$

The above result corresponds to Theorem 8.5 within the text and supplies the explicit description of the ring $B_{r}$ as a module over the Lie algebra $g l\left(\bigwedge^{k} V\right)$, for all $k \geq 0$. In fact, the $\mathcal{E}_{\boldsymbol{\mu}, \boldsymbol{v}}^{k}$-image of $S_{\lambda}$ is determined by the coefficient of $s_{\boldsymbol{\mu}}\left(\mathbf{z}_{k}\right) s_{\boldsymbol{v}}\left(\mathbf{w}_{k}^{-1}\right)$ obtained by the expansion of the right hand side of (2). This may sounds tricky to evaluate, but is nothing else than the coefficient of

$$
z_{1}^{k-1+\mu_{1}} \cdots z_{k}^{\mu_{k}} \cdot w_{1}^{-k+1-v_{1}} \cdots w_{k}^{-v_{k}}
$$

of the right hand side of (2), multiplied by the Vandermonde determinants of $\mathbf{z}_{k}$ and $\mathbf{w}_{k}^{-1}$.

That $B_{r}$ is a representation of $g l\left(\bigwedge^{k} V\right)$ is easy to see in very special cases. For $k=0$, it is the multiplication by rational numbers, as $\bigwedge^{0} V=\mathbb{Q}$, while for $k>r$ is the trivial null representation. The case $r=k=1$ recovers the well known general fact that every vector space is a module over the Lie algebra of its own endomorphisms. In fact the linear extension of the set map $e_{1}^{i} \mapsto b_{i}$ is a vector space isomorphism $B_{1} \rightarrow V$, making $B_{1}$ into a $g l(V)$ module, by pulling back that structure from $V$. Our Main Theorem then takes into account the general case.

\subsection{The boson-fermion correspondence and the DJKM representation}

The $g l\left(\bigwedge^{k} V\right)$-module structure of $B_{r}$, described in Main Theorem, will be referred to as bosonic representation of $g l\left(\bigwedge^{k} V\right)$, by a possibly strong, but suggestive, abuse of terminology, due to the evident relationship with the pioneering work by Date, Jimbo, Kashiwara and Miwa (DJKM) [8] (see also [24, 25]) which also fits into the more general framework considered in the reference [7]. 
As a matter of fact, one main motivation of this paper was to better understand a fundamental, although elementary, representation theoretical fact. Let $\mathcal{V}:=\bigoplus_{j \in \mathbb{Z}} \mathbb{Q} \cdot b_{j}$ be a vector space with basis $\mathbf{b}:=\left(b_{j}\right)_{j \in \mathbb{Z}}$, parameterized by the integers (one may think of $\mathcal{V}$ as being the vector space $\mathbb{Q}\left[X^{-1}, X\right]$ of the Laurent polynomials) and $\mathcal{V}^{*}$ its restricted dual with basis $\left(\beta_{j}\right)_{j \in \mathbb{Z}}$. It is well known that $\mathcal{V} \oplus \mathcal{V}^{*}$ supports a canonical structure of Clifford algebra $\mathcal{C}:=\mathcal{C}\left(\mathcal{V} \oplus \mathcal{V}^{*}\right)([9$, p. 85] or [18]) and that the Fermionic Fock space $F$ (also called the semi-infinite wedge power and denoted by $\bigwedge^{\infty / 2} \mathcal{V}$ ) is an irreducible representation of $\mathcal{C}$. More precisely, $F$ is an invertible module over the Lie super-algebra $\mathcal{C}$ generated by a distinguished vector $|0\rangle$, the vacuum, that in the formalism of the infinite wedge power can be suggestively written as $b_{0} \wedge b_{-1} \wedge b_{-2} \wedge \cdots$

The huge Clifford algebra $\mathcal{C}$, whose elements are finite linear combinations of words of the form $b_{i_{i}} \cdots b_{i_{h}} \beta_{j_{1}} \cdots \beta_{j_{k}}$, contains in a natural way all, but not only, the Lie algebras $g l\left(\bigwedge^{k} V\right)$, for all $k \geq 0$. In particular, it turns out that $F$ is a $g l\left(\bigwedge^{k} \mathcal{V}\right)$-module for all $k \geq 0$. Then, the bosonic Fock space $B:=B_{\infty}:=\mathbb{Q}\left[e_{1}, e_{2}, \ldots\right]$ gets a $g l\left(\bigwedge^{k} V\right)$-module structure, for all $k \geq 0$, pulled back from $F$ via the boson-fermion correspondence, a natural module isomorphism $B \rightarrow F$ over the infinite dimensional Lie Heisenberg algebra. The latter may well be interpreted as a sort of Poincaré duality for infinite dimensional Grassmannians. The case $k=1$ recovers precisely the celebrated bosonic vertex operator representation of the Lie algebra $g l_{\infty}(\mathbb{Q}):=g l(\mathcal{V})=g l\left(\bigwedge^{1} V\right)$ due to Date, Jimbo, Kashiwara and Miwa ([8, 24]) as in e.g. [25, Theorem 5.1] we have alluded above.

Our paper, however, aims to look at more traditional, but relevant, contexts. Exactly as in the case of the Fermionic Fock space $\mathcal{F}$, the exterior algebra of $V \cong \mathbb{Q}[X]$ is an irreducible representation of the canonical Clifford algebra $\mathcal{C}$ supported on $V \oplus V^{*}$. This occurrence convinced ourselves to give a closer look to the $g l(\bigwedge V)$-structure of $\bigwedge V$, certainly not treated in any literature we have consulted up to now.

We have so gotten a description of the $g l\left(\bigwedge^{k} V\right)$-module structure of $B_{r}$, which generalises the case $r<\infty$ and $k=1$ studied in [20]. The output is that the direct sum $\bigoplus_{k \geq 0} g l\left(\bigwedge^{k} V\right)$ is a Lie subalgebra of $g l(\bigwedge V)$, represented by $B_{r}$ for all $r \geq 0$. In the case of the fermionic Fock space, the $g l\left(\bigwedge^{1} V\right)$-structure of $B_{\infty}$ is the DJKM one [8, 24]. The general case, which amounts to the description of the $g l(\bigwedge \mathcal{V})$-module structure of $\mathcal{F}$, is faced in the contribution [2] as a best example of the extension of the techniques used in [21].

\subsection{Methods and their applications}

The vertex operators occurring in our description of $B_{r}$ as a representation of $g l\left(\bigwedge^{k} V\right)$ are defined by means of Schubert derivations, which are distinguished Hasse-Schmidt (HS) derivations on exterior algebras.

HS derivations were first introduced in [13] and extensively treated in [18]; see also the survey [1] or [6, p. 116], for more discussions. In a finite dimensional context Schubert derivations are related to Chern and Segre polynomials of the tautological bundle over a Grassmannian. The point is that the Segre and Chern polynomials act as a HS-derivation on the exterior algebra of the homology of the projective space, which is the same as saying that to do Schubert calculus on Grassmannians, Bézout theorem suffices.

Hasse-Schmidt derivations on exterior algebras have shown their versatility in applications to improve effectiveness in Schubert Calculus computations (see $[4,5]$ ), to equivariant cohomology of Grassmannians (Cf. [22], but also [26]), to generalise the Cayley-Hamilton theorem [16, 23], with perspective applications to globalise the local Wronskian as in $[15$, Section 4.2$]$, or, inspired by $[14,17]$, like in $[2,20,21]$ and in the present paper, to 
revisit the bosonic vertex representation of Lie algebras of endomorphisms as in [8] (see also [24] and [25, Propositions 5.2-5.3]), providing new methods and new insight.

The Schubert derivations we introduce here, denoted by $\sigma_{+}(z), \bar{\sigma}_{+}(z), \sigma_{-}(w)$ and $\bar{\sigma}_{-}(w)$ enjoy some nice commutation rules. Those with the same sign as subscripts commute in the algebra of endomorphisms of the exterior algebra. However, due to the fact that $\sigma_{-}(w)$ and $\bar{\sigma}_{-}(w)$ are locally nilpotent, they commute with $\sigma_{+}(z)$ and $\bar{\sigma}_{+}(z)$ only up to the multiplication by a rational function.

\subsection{Organization of the paper}

To be as much self-contained as possible, we collect most of preliminaries and basic notation in Sect. 2. The first part recalls basics of the theory of symmetric polynomials as, e.g., in [12]. The second part accounts for the invertible Hasse-Schmidt derivation on an exterior algebra, essential in the subsequent sections.

Section 3 contains the explicit expression of the Schubert derivations that makes evident their strong connection with vertex operators.

Section 4 also contains an effective definition of what we have proposed to name vertex operators on a Grassmann algebra, because an obvious relationship with those occurring in the classical boson-fermion correspondence. Also, to check the Main Theorem without neglecting any minimum detail, we state and prove in Sect. 4 relevant commutation rules, some of which can be recognized within the phrasing of the categorical framework for the boson-fermion correspondence, depicted in [10] (see also [28] for a recent update).

In Sect. 5 we instead study commutation rules involving the contraction operator: this is a typical issue in the situation involving a finite wedge power. In fact, the finiteness makes the subject trickier than when working with the infinite wedge power.

Vertex operators in the sense of Definition 4.3 are homogeneous operators on the exterior algebra, one of positive and the other of negative degree. We devote one section to each one of them (Sects. 7 and 8) to dig up their relationship with basic computations in multilinear algebra, such as wedging and contracting.

Certainly this idea is already present in the infinite wedge power context (e.g. [25, Chapter 5]), but the present article, together with [19-21], is the first instance of applications of the techniques and ideas in finite dimensional landscapes.

Finally, last Sect. 8 is concerned with the proof of the Main Theorem together with some of its straightforward declinations in terms of certain familiar objects, like suitable deformations of the same Giambelli's determinants occurring in classical Schubert Calculus, see Theorem 8.10. To achieve the proof of the Main Theorem, some preliminary lemmas (such as 8.2 and 8.3) are proved. We believe that these lemmas along with Theorems 6.5 and 7.3, are interesting in their own, as pieces of multilinear algebra properties addressed to wider general mathematical audiences.

\section{Preliminaries and notation}

\subsection{Partitions}

A partition is a monotonic non increasing sequence $\lambda:=\left(\lambda_{1} \geq \lambda_{2} \geq \ldots\right)$ of non negative integers, said to be its parts. The length $\ell(\lambda)$ is the number of its non zero parts, and $|\lambda|=\sum_{i \geq 0} \lambda_{i}$ is its weight. We denote by $\mathcal{P}_{r}$ be the set of all partitions of length at most $r$. 


\subsection{Exterior powers, exterior algebras and duality pairing}

Let $V:=\bigoplus_{i \geq 0} \mathbb{Q} \cdot b_{i}$ be the vector space with basis $\mathbf{b}:=\left(b_{i}\right)_{i \geq 0}$. The restricted dual of $V$ is $V^{*}:=\bigoplus_{j \geq 0} \mathbb{Q} \cdot \beta_{j}$, where $\beta_{j}\left(b_{i}\right)=\delta_{i j}$. Denote by $\mathbf{b}(z)$ and $\boldsymbol{\beta}\left(w^{-1}\right)$ the generating series of the basis elements of $V$ and of $V^{*}$ respectively, i.e.:

$$
\mathbf{b}(z):=\sum_{i \geq 0} b_{i} z^{i} \quad \text { and } \quad \boldsymbol{\beta}\left(w^{-1}\right):=\sum_{j \geq 0} \beta_{j} w^{-j} .
$$

The exterior algebra of $V$ is $\bigwedge V:=\bigoplus_{j \geq 0} \bigwedge^{j} V$, the direct sum of the exterior powers $\bigwedge^{j} V$, where $\bigwedge^{0} V=\mathbb{Q}$ and $\bigwedge^{1} V=V$. The algebra structure is given by the $\mathbb{Q}$-linear extension of the juxtaposition. To each $\lambda \in \mathcal{P}_{r}$ we associate

$$
[\mathbf{b}]_{\lambda}^{r}:=b_{r-1+\lambda_{1}} \wedge b_{r-2+\lambda_{2}} \wedge \cdots \wedge b_{\lambda_{r}} \in \bigwedge^{r} V
$$

so that $\left([\mathbf{b}]_{\lambda}^{r}\right)_{\lambda \in \mathcal{P}_{r}}$ is a $\mathbb{Q}$-basis of $\bigwedge^{r} V$. The pairing

$$
\left(\beta_{i_{1}} \wedge \cdots \wedge \beta_{i_{r}}\right)\left(v_{1} \wedge \cdots \wedge v_{r}\right)=\left|\begin{array}{ccc}
\beta_{i_{1}}\left(v_{1}\right) & \cdots & \beta_{i_{1}}\left(v_{r}\right) \\
\vdots & \ddots & \vdots \\
\beta_{i_{r}}\left(v_{1}\right) & \cdots & \beta_{i_{r}}\left(v_{r}\right)
\end{array}\right|
$$

establishes a natural identification between $\bigwedge^{r} V^{*}$ and $\left(\bigwedge^{r} V\right)^{*}$. If one denotes by $[\boldsymbol{\beta}]_{\mu}^{r}$ the basis element

$$
\beta_{r-1+\mu_{1}} \wedge \cdots \wedge \beta_{\mu_{r}}
$$

of $\bigwedge^{r} V^{*}$, an easy check shows that $[\boldsymbol{\beta}]_{\mu}^{r}\left([\mathbf{b}]_{\lambda}^{r}\right)=\delta_{\mu, \lambda}$. The pairing (5) enables to attach to any $\beta \in V^{*}$ a map $\left.\beta\right\lrcorner: \bigwedge V \rightarrow \bigwedge V$ of degree -1 (with respect to the graduation of the exterior algebra) via the equality

$$
\eta(\beta\lrcorner u)=(\beta \wedge \eta)(u), \quad \forall(u, \eta) \in \bigwedge^{r} V \times \bigwedge^{r-1} V^{*}
$$

\subsection{The ring $B_{r}$}

Let $r \geq 1$. The main character of this paper is the polynomial ring $B_{r}:=\mathbb{Q}\left[e_{1}, \ldots, e_{r}\right]$ in the $r \geq 1$ indeterminates $\left(e_{1}, \ldots, e_{r}\right)$ (by convention $B_{0}=\mathbb{Q}$ ).

Given the generic polynomial $E_{r}(z):=1-e_{1} z+\cdots+(-1)^{r} e_{r} z^{r} \in B_{r}[z]$, one considers the sequence $H_{r}:=\left(h_{j}\right)_{j \in \mathbb{Z}}$ defined by the equality:

$$
\sum_{n \in \mathbb{Z}} h_{n} z^{n}:=\frac{1}{E_{r}(z)} .
$$

holding in $B_{r}[z]$. In particular $h_{j}=0$ if $j<0$ and $h_{0}=1$. Moreover for $j \geq 0$, the term $h_{j}$ is an explicit polynomial in $\left(e_{1}, \ldots, e_{r}\right)$, homogeneous of degree $j$, once one gives weight $i$ to $e_{i}$. The Schur determinants 


$$
\Delta_{\lambda}\left(H_{r}\right):=\operatorname{det}\left(h_{\lambda_{j}-j+i}\right)_{1 \leq i, j \leq r}=\left|\begin{array}{cccc}
h_{\lambda_{1}} & h_{\lambda_{2}-1} & \ldots & h_{\lambda_{r}-r+1} \\
h_{\lambda_{1}+1} & h_{\lambda_{2}} & \ldots & h_{\lambda_{r}-r+2} \\
\vdots & \vdots & \ddots & \vdots \\
h_{\lambda_{1}+r-1} & h_{\lambda_{2}+r-2} & \ldots & h_{\lambda_{r}}
\end{array}\right|,
$$

form a $\mathbb{Q}$-basis of $B_{r}$ parametrized by the partitions of length at most $r$ :

$$
B_{r}:=\bigoplus_{\lambda \in \mathcal{P}_{r}} \mathbb{Q} \cdot \Delta_{\lambda}\left(H_{r}\right)
$$

It follows that $B_{r}$ is naturally isomorphic to $\bigwedge^{r} V$ via the $\mathbb{Q}$-linear extension of the sets map

$$
\Delta_{\lambda}\left(H_{r}\right) \mapsto[\mathbf{b}]_{\lambda}^{r} .
$$

\subsection{Schur polynomials}

Especially in the last section we shall be concerned with Schur polynomials in a set of indeterminates. We recall them here. For each partition of length at most $k$ and any set of $k$ formal variables $\mathbf{x}_{k}:=\left(x_{1}, \ldots, x_{k}\right)$, one defines

$$
\Delta_{\lambda}\left(\mathbf{x}_{k}\right)=\operatorname{det}\left(x_{j}^{\lambda_{k-i+1}+i-1}\right)=\left|\begin{array}{cccc}
x_{1}^{\lambda_{k}} & x_{2}^{\lambda_{k}} & \cdots & x_{k}^{\lambda_{k}} \\
x_{1}^{1+\lambda_{k-1}} & x_{2}^{1+\lambda_{k-1}} & \cdots & x_{k}^{1+\lambda_{k-1}} \\
\vdots & \vdots & \ddots & \vdots \\
x_{1}^{k-1+\lambda_{1}} & x_{2}^{k-1+\lambda_{1}} & \cdots & x_{k}^{k-1+\lambda_{1}}
\end{array}\right| .
$$

This is an skew symmetric polynomials in $\left(x_{1}, \ldots, x_{k}\right)$ and therefore divisible by the Vandermonde determinant

$$
\Delta_{0}\left(\mathbf{x}_{k}\right):=\left|\begin{array}{cccc}
1 & 1 & \cdots & 1 \\
x_{1} & x_{2} & \cdots & x_{k} \\
\vdots & \vdots & \ddots & \vdots \\
x_{1}^{k-1} & x_{2}^{k-1} & \cdots & x_{k}^{k-1}
\end{array}\right|=\prod_{i<j}\left(x_{j}-x_{i}\right)
$$

The Schur polynomial associated to $\mathbf{x}_{k}$ and the partition $\lambda$ is defined by the equality

$$
\Delta_{\lambda}\left(\mathbf{x}_{k}\right)=s_{\lambda}\left(\mathbf{x}_{k}\right) \cdot \Delta_{0}\left(\mathbf{x}_{k}\right),
$$

often said to be the Jacobi-Trudy formula.

\subsection{Hasse-Schmidt derivations on exterior algebras}

Let now $\bigwedge V[z]$ denote the formal power series in the indeterminate $z$ with coefficients in the exterior algebra $\bigwedge V$ of $V$. If $\mathcal{S}$ is any set of indeterminates over $\mathbb{Q}$, denote by $\mathbb{Q}[\mathcal{S}]$ the corresponding algebra of formal power series. The following is an extended reformulation of the main definition of the reference [13] (see also [18]). By a Hasse-Schmidt derivation on $\bigwedge V$ we mean any $\mathbb{Q} \llbracket \mathcal{S} \rrbracket$-linear extension of a $\mathbb{Q}$-linear map $\mathcal{D}(z): \bigwedge V \rightarrow \wedge V \llbracket z \rrbracket$ such that

$$
\mathcal{D}(z)(u \wedge v)=\mathcal{D}(z) u \wedge \mathcal{D}(z) v, \quad \forall u, v \in \bigwedge V
$$


which, by abuse of notation, will be denoted by the same symbol

$$
\mathcal{D}(z): \mathbb{Q} \llbracket \mathcal{S} \rrbracket \otimes_{\mathbb{Q}} \bigwedge V \rightarrow \mathbb{Q} \llbracket \mathcal{S} \rrbracket \otimes_{\mathbb{Q}} \bigwedge V \llbracket z \rrbracket,
$$

(instead of the more precise, but lengthier, $1_{\mathbb{Q}[\mathcal{S}]} \otimes_{\mathbb{Q}} \mathcal{D}(z)$ ).

If $\mathcal{D}_{i} \in \operatorname{End}_{\mathbb{Q}}(\bigwedge V)$ are such that $\sum_{i \geq 0} \mathcal{D}_{i} z^{i}:=\mathcal{D}(z)$, then (12) is equivalent to the system of relations

$$
\mathcal{D}_{i}(u \wedge v)=\sum_{j=0}^{i} \mathcal{D}_{j} u \wedge D_{i-j} v, \quad(i \geq 0)
$$

The notation

$$
\mathcal{D}(z)[\mathbf{b}]_{\lambda}^{r}=[\mathcal{D}(z) \mathbf{b}]_{\lambda}^{r}
$$

will be used as a shorthand for the equality

$$
\mathcal{D}(z)[\mathbf{b}]_{\lambda}^{r}=\mathcal{D}(z)\left(b_{r-1+\lambda_{1}} \wedge \cdots \wedge b_{\lambda_{r}}\right)=\mathcal{D}(z) b_{r-1+\lambda_{1}} \wedge \cdots \wedge \mathcal{D}(z) b_{\lambda_{r}}
$$

meaning that $\mathcal{D}(z)$ is a HS-derivation. By [23, Proposition 3.3], if $\mathcal{D}_{0}$ is invertible in $\operatorname{End}_{\mathbb{Q}}(\bigwedge V)$, then $\mathcal{D}(z)$ is invertible as a $\operatorname{End}_{\mathbb{Q}}(\bigwedge V)$-valued formal power series and its inverse, $\overline{\mathcal{D}}(z)$, is an HS-derivation as well.

2.6 Proposition Let $\mathcal{D}_{0}$ be invertible in $\operatorname{End}_{\mathbb{Q}}(\bigwedge V)$. Then the integration by parts formulas follow for all $u, v \in \bigwedge V$ :

$$
\begin{aligned}
& \mathcal{D}(z)(\overline{\mathcal{D}}(z) u \wedge v)=u \wedge \mathcal{D}(z) v, \\
& \overline{\mathcal{D}}(z)(\mathcal{D}(z) u \wedge v)=u \wedge \overline{\mathcal{D}}(z) v .
\end{aligned}
$$

Formulas (14) and (15) are implicitly assuming the $\mathbb{Q} \llbracket z \rrbracket$-linearity of $\mathcal{D}(z)$ we alluded to in Definition 2.5. The extension of the linearity of HS-derivations over polynomial algebras will be assumed in the following without any further mention.

\subsection{Transposition}

The transpose $\mathcal{D}(z)^{T}: \bigwedge V^{*} \rightarrow \bigwedge V^{*}[[z]]$ of the HS derivation $\mathcal{D}(z)$ is defined via its action on homogeneous elements. For $\eta \in \bigwedge^{r} V^{*}$, one stipulates that $\mathcal{D}(z)^{T} \eta(u)=\eta(\mathcal{D}(z) u)$, for all $u \in \bigwedge^{r} V$. By [19, Proposition 2.8] $\mathcal{D}(z)^{T}$ is a HS-derivation of $\bigwedge V^{*}$. 


\section{Recap on Schubert derivations}

\subsection{Exterior algebras representations of endomorphisms}

Consider the natural representation $\delta: \operatorname{End}(V) \rightarrow \operatorname{End}(\bigwedge V)$ making any $\phi \in \operatorname{End}(V)$ into an (even) derivation $\delta(\phi)$ of $\bigwedge V$. In other words, $\delta(\phi)$ is the unique $\mathbb{Q}$-vector space endomorphism of $\bigwedge V$ such that

$$
\delta(\phi)(v \wedge w)=\delta(\phi) v \wedge w+v \wedge \delta(\phi) w
$$

for all $v, w \in \bigwedge V$, together with the initial condition $\delta(\phi) u=\phi(u)$ holding for all $u \in V=\bigwedge^{1} V$. An easy check shows that

$$
\mathcal{D}^{\phi}(z)=\exp \left(\sum_{i \geq 1} \frac{1}{i} \delta\left(\phi^{i}\right) z^{i}\right),
$$

is the unique HS derivation on $\bigwedge V$ such that $\mathcal{D}^{\phi}(z)_{\left.\right|_{V}}=\sum_{i \geq 0} \phi^{i} z^{i}$.

Let now $\sigma_{1}: V \rightarrow V$ be such that $\sigma_{1} b_{j}=b_{j+1}$ and $\sigma_{-1}: V \rightarrow V$ such that $\sigma_{-1} b_{j}=b_{j-1}$, where by convention we put $b_{k}=0$ if $k<0$.

3.2 Definition The Schubert derivations on $\bigwedge V$ are the HS-derivations $\sigma_{+}(z): \bigwedge V \rightarrow \bigwedge V \llbracket z \rrbracket$ and $\sigma_{-}(z): \bigwedge V \rightarrow \bigwedge V\left[z^{-1}\right]$ defined by

$$
\begin{gathered}
\sigma_{+}(z)=\sum_{i \geq 0} \sigma_{i} z^{i}:=\exp \left(\sum_{i \geq 1} \frac{1}{i} \delta\left(\sigma_{1}^{i}\right) z^{i}\right), \\
\sigma_{-}(z)=\sum_{i \geq 0}(-1)^{i} \sigma_{-i} z^{-i}:=\exp \left(\sum_{i \geq 1} \frac{1}{i} \delta\left(\sigma_{-1}^{i}\right) z^{-i}\right),
\end{gathered}
$$

and their inverses in $\operatorname{End}_{\mathbb{Q}}(\bigwedge V) \llbracket z \rrbracket$ and $\operatorname{End}_{\mathbb{Q}}(\bigwedge V)\left[z^{-1}\right]$ respectively:

$$
\begin{gathered}
\bar{\sigma}_{+}(z)=\sum_{i \geq 0}(-1)^{i} \bar{\sigma}_{i} z^{i}:=\exp \left(-\sum_{i \geq 1} \frac{1}{i} \delta\left(\sigma_{1}^{i}\right) z^{i}\right), \\
\bar{\sigma}_{-}(z)=\sum_{i \geq 0}(-1)^{i} \bar{\sigma}_{-i} z^{-i}:=\exp \left(-\sum_{i \geq 1} \frac{1}{i} \delta\left(\sigma_{-1}^{i}\right) z^{-i}\right) .
\end{gathered}
$$

In particular:

$$
\bar{\sigma}_{ \pm}(z) u=u-\sigma_{ \pm 1} u \cdot z^{ \pm 1}, \quad \forall u \in V=\bigwedge^{1} V
$$

3.3 Remark It is easily seen that $\sigma_{ \pm}(z)$ and $\bar{\sigma}_{ \pm}(z)$ are the unique HS-derivations on $\bigwedge V$ such that

$$
\sigma_{+}(z) b_{j}=\sum_{i \geq 0} b_{j+i} z^{i}, \quad \bar{\sigma}_{+}(z) b_{j}=b_{j}-b_{j+1} z
$$


and

$$
\sigma_{-}(z) b_{j}=\sum_{i \geq 0} \frac{b_{j-i}}{z^{i}}, \quad \bar{\sigma}_{-}(z) b_{j}=b_{j}-\frac{b_{j-1}}{z},
$$

putting $b_{i}=0$ for $i<0$.

\section{$3.2 B_{r}$-module structure of $\bigwedge^{r} V$}

We exploit the Schubert derivation $\bar{\sigma}_{+}(z)$ or, equivalently, its inverse $\sigma_{+}(z)$, to endow $\bigwedge^{r} V$ with a $B_{r}$-module structure, by declaring that $e_{i} u=\bar{\sigma}_{i} u$ or, equivalently, $h_{i} \mathbf{u}=\sigma_{i} \mathbf{u}$, for all $\mathbf{u} \in \bigwedge V$. In particular:

$$
\bar{\sigma}_{+}(z) \mathbf{u}=E_{r}(z) \cdot \mathbf{u} \quad \text { and } \quad \sigma_{+}(z) \mathbf{u}:=\frac{1}{E_{r}(z)} \mathbf{u}, \quad \forall \mathbf{u} \in \bigwedge^{r} V .
$$

The fact that such a product structure is compatible with the natural vector space isomorphism $B_{r} \rightarrow \bigwedge^{r} V$ given by (11) is a consequence of

3.5 Proposition Giambelli's formula for the Schubert derivation $\sigma_{+}(z)$ holds:

$$
[\mathbf{b}]_{\lambda}^{r}=\Delta_{\lambda}\left(\sigma_{+}(z)\right)[\mathbf{b}]_{0}^{r}:=\operatorname{det}\left(\sigma_{\lambda_{j}-j+i}\right)_{1 \leq i, j \leq r}[\mathbf{b}]_{0}^{r} .
$$

Hence $\bigwedge^{r} V$ is a free $B_{r}$-module of rank 1 generated by $[\mathbf{b}]_{0}^{r}$.

Proof Formula (22) may be inferred as a particular case of the general determinantal formula for the exterior power of a polynomial ring due to Laksov and Thorup as in [27, Main Theorem 0.1]. It follows that $[\mathbf{b}]_{\lambda}^{r}=\Delta_{\lambda}\left(\sigma_{+}(z)\right)[\mathbf{b}]_{0}^{r}=\Delta_{\lambda}\left(H_{r}\right)[\mathbf{b}]_{0}^{r}$, proving the second part of the claim.

By virtue of 3.5 , the set map $\Delta_{\lambda}\left(H_{r}\right) \mapsto \Delta_{\lambda}\left(H_{r}\right)[\mathbf{b}]_{0}^{r}$ extends to a well defined vector space isomorphisms $B_{r} \rightarrow \bigwedge^{r} V$, as it maps the basis $\left(\Delta_{\lambda}\left(H_{r}\right)\right)_{\lambda \in \mathcal{P}_{r}}$ of $B_{r}$ to the basis $\left([\mathbf{b}]_{\lambda}^{r}\right)_{\lambda \in \mathcal{P}_{r}}$ of $\bigwedge^{r} V$. The fact that $\bigwedge^{r} V$ is a free $B_{r}$-module of rank 1 generated by $[\mathbf{b}]_{0}^{r}$, as prescribed by equality (22), shows that the Schubert derivations $\sigma_{-}(z), \bar{\sigma}_{-}(z)$ induce maps $B_{r} \rightarrow B_{r}\left[z^{-1}\right]$ which, abusing notation, will be denoted in the same way. Their action on a basis element $\Delta_{\lambda}\left(H_{r}\right)$ of $B_{r}$ is defined through its action on $\bigwedge^{r} V$ :

$$
\begin{gathered}
\left(\bar{\sigma}_{-}(z) \Delta_{\lambda}\left(H_{r}\right)\right)[\mathbf{b}]_{0}^{r}=\bar{\sigma}_{-}(z)[\mathbf{b}]_{\lambda}^{r}, \\
\left(\sigma_{-}(z) \Delta_{\lambda}\left(H_{r}\right)\right)[\mathbf{b}]_{0}^{r}=\sigma_{-}(z)[\mathbf{b}]_{\lambda}^{r} .
\end{gathered}
$$

Denote by $\bar{\sigma}_{-}(z) H_{r}$ (respectively $\left.\sigma_{-}(z) H_{r}\right)$ the sequence $\left(\bar{\sigma}_{-}(z) h_{j}\right)_{j \in \mathbb{Z}}$ (respectively $\left.\left(\sigma_{-}(z) h_{j}\right)_{j \in \mathbb{Z}}\right)$. By using [19, Theorem 5.7], and exploiting the Laksov \& Thorup determinantal formula as in [27, Main Theorem 0.1], one obtains the following statement, which gives a practical way to evaluate the image of $\Delta_{\lambda}\left(H_{r}\right)$ through the maps $\bar{\sigma}_{-}(z)$ and $\sigma_{-}(z)$ defined by (23) and (24).

3.6 Proposition ([19, Proposition 5.3]). For all $r \geq 0$ and all $\lambda \in \mathcal{P}_{r}$ 


$$
\sigma_{-}(z) h_{j}=\sum_{i \geq 0} \frac{h_{j-i}}{z^{i}} \quad \text { and } \quad \bar{\sigma}_{-}(z) h_{j}=h_{j}-\frac{h_{j-1}}{z}
$$

Moreover:

$$
\sigma_{-}(z) \Delta_{\lambda}\left(H_{r}\right)=\Delta_{\lambda}\left(\sigma_{-}(z) H_{r}\right) \quad \text { and } \quad \bar{\sigma}_{-}(z) \Delta_{\lambda}\left(H_{r}\right)=\Delta_{\lambda}\left(\bar{\sigma}_{-}(z) H_{r}\right) .
$$

3.7 Remark It is important to notice that (26) only holds if $\ell(\lambda) \leq r$. For example

$$
\Delta_{(1,1)}\left(\bar{\sigma}_{-}(z) H_{1}\right)=\left|\begin{array}{cc}
h_{1}-\frac{1}{z} & 1 \\
h_{2}-\frac{h_{1}}{z} & h_{1}-\frac{1}{z}
\end{array}\right|=-\frac{h_{1}}{z}+\frac{1}{z^{2}} \neq 0=\bar{\sigma}_{-}(z) \Delta_{(1,1)}\left(H_{1}\right) .
$$

\section{Commutation rules for Schubert derivations}

\subsection{Product of Schubert derivations}

For $k \geq 1$, let $\mathbf{z}_{k}$ denote the ordered $k$-tuple $\left(z_{1}, \ldots, z_{k}\right)$ of formal variables. By $\mathbf{z}_{k}^{-1}$ we shall mean the $k$-tuple of the formal inverses $\left(z_{1}^{-1}, \ldots, z_{k}^{-1}\right)$. Define maps $\left.\sigma_{ \pm}\left(\mathbf{z}_{k}\right), \bar{\sigma}_{ \pm}\left(\mathbf{z}_{k}\right): \bigwedge V \rightarrow \bigwedge V \llbracket \mathbf{z}_{k}, \mathbf{z}_{k}^{-1}\right]$ respectively by

$$
\sigma_{ \pm}\left(\mathbf{z}_{k}\right):=\sigma_{ \pm}\left(z_{1}\right) \cdots \sigma_{ \pm}\left(z_{k}\right) \quad \text { and } \quad \bar{\sigma}_{ \pm}\left(\mathbf{z}_{k}\right):=\bar{\sigma}_{ \pm}\left(z_{1}\right) \cdots \bar{\sigma}_{ \pm}\left(z_{k}\right) \text {. }
$$

The maps occurring in formulas (27) are multivariate HS derivations on $\bigwedge V$, in the sense that, for instance, $\sigma_{+}\left(\mathbf{z}_{k}\right)(u \wedge v)=\sigma_{+}\left(\mathbf{z}_{k}\right) u \wedge \sigma_{+}\left(\mathbf{z}_{k}\right) v$, as it is easy to check and adopting the linear extension of the Schubert derivation to polynomial coefficients according to Definition 2.5. The same holds verbatim for $\sigma_{-}\left(\mathbf{z}_{k}\right)$ and $\bar{\sigma}_{ \pm}\left(\mathbf{z}_{k}\right)$. It is an important point that the multivariate HS derivations in (27) are symmetric in the formal variables $z_{i}$ and $w_{i}$. This is a consequence of the first of the commutation rules obeyed by the product of Schubert derivations listed in this section and to be used in the sequel.

4.2 Proposition Let $z, w$ be arbitrary formal variables. The equalities

$$
\begin{aligned}
& \bar{\sigma}_{ \pm}(z) \bar{\sigma}_{ \pm}(w)=\bar{\sigma}_{ \pm}(w) \bar{\sigma}_{ \pm}(z), \\
& \sigma_{ \pm}(z) \sigma_{ \pm}(w)=\sigma_{ \pm}(w) \sigma_{ \pm}(z),
\end{aligned}
$$

hold in $\operatorname{End}_{\mathbb{Q}}(\bigwedge V) \llbracket z^{ \pm 1}, w^{ \pm 1} \rrbracket$.

Proof Formulas (28) and (29) are obvious consequences of the fact that if $i, j \geq 0$, then $\sigma_{ \pm i}$ and $\sigma_{ \pm j}$ are pairwise commuting. It is sufficient, then, to show that they commute when restricted to $V$, because if they do, then

$$
\sigma_{ \pm}(z) \sigma_{ \pm}(w)[\mathbf{b}]_{\lambda}^{r}=\left[\sigma_{ \pm}(z) \sigma_{ \pm}(w) \mathbf{b}\right]_{\lambda}^{r}=\left[\sigma_{ \pm}(w) \sigma_{ \pm}(z) \mathbf{b}\right]_{\lambda}^{r}
$$


having used notation as in (13). But $\sigma_{ \pm i} \sigma_{ \pm j} u=\sigma_{ \pm 1}^{i+j} u=\sigma_{ \pm j} \sigma_{ \pm i} u$ for all $u \in V$, and then the claim follows.

In order to give a compact expression of the $g l\left(\bigwedge^{k} V\right)$-module structure of $B_{r}$, we shall need to introduce a generalisation of the classical vertex operators arising in the context of the so-called boson-fermion correspondence, like in e.g. [25]. We look at it as a generalisation of the isomorphism $B_{r} \rightarrow \bigwedge^{r} V$, recalled in Sect. 2.1, reaffirmed and refined in Proposition 3.5.

4.3 Definition By vertex operators on $\bigwedge V$ we mean the $\mathbb{Q}\left[\mathbf{z}_{k}, \mathbf{z}_{k}^{-1}\right]$-linear maps $\Gamma\left(\mathbf{z}_{k}\right), \Gamma^{*}\left(\mathbf{z}_{k}\right): \bigwedge V \rightarrow(\bigwedge V)\left[\mathbf{z}_{k}, \mathbf{z}_{k}^{-1}\right]$ of degree 1 and -1 , with respect to the exterior algebra graduation, given by:

$$
\begin{gathered}
\Gamma\left(\mathbf{z}_{k}\right)[\mathbf{b}]_{\lambda}^{r}=\sigma_{+}\left(\mathbf{z}_{k}\right) \bar{\sigma}_{-}\left(\mathbf{z}_{k}\right)[\mathbf{b}]_{\lambda}^{r+k}, \\
\Gamma^{*}\left(\mathbf{z}_{k}\right)[\mathbf{b}]_{\lambda}^{r}=\left(\bar{\sigma}_{+}\left(\mathbf{z}_{k}\right) \Delta_{\lambda}\left(\sigma_{-}\left(\mathbf{z}_{k}\right) H_{r-k}\right)\right)[\mathbf{b}]_{0}^{r-k} .
\end{gathered}
$$

Proposition 4.2 guarantees that the vertex operators $\Gamma\left(\mathbf{z}_{k}\right)$ and $\Gamma^{*}\left(\mathbf{z}_{k}\right)$ are symmetric in the formal variables $\left(z_{1}, \ldots, z_{k}\right)$. They will be studied in a more detailed way in Sects. 6 and 7 , exploiting further commutation relations, for which we need the preliminary work exposed below. As a matter of fact, we notice that the commutativity of the product of Schubert derivations is granted only if they are of the same kind (both subscripts "+" or both subscripts "-"). In general, for $i, j>0, \sigma_{i}$ and $\sigma_{-j}$ do not commute, because $\sigma_{-j}$ is locally nilpotent. The simplest example is: $\sigma_{-1} \sigma_{1} b_{0}=b_{0} \neq 0=\sigma_{1} \sigma_{-1} b_{0}$. The general pattern is that commutativity only holds up to the multiplication by a rational function.

\subsection{Proposition}

i) If $\lambda \in \mathcal{P}_{r} \backslash \mathcal{P}_{r-1}($ i.e. $\ell(\lambda)=r)$, then $\bar{\sigma}_{-}(w)$ commutes with both $\sigma_{+}(z)$ and $\bar{\sigma}_{+}(z)$, i.e.

$$
\bar{\sigma}_{-}(w) \sigma_{+}(z)=\sigma_{+}(z) \bar{\sigma}_{-}(w),
$$

and

$$
\bar{\sigma}_{-}(w) \bar{\sigma}_{+}(z)=\bar{\sigma}_{+}(z) \bar{\sigma}_{-}(w) .
$$

ii) if $\lambda \in \mathcal{P}_{r-1}$ (i.e. $\left.[\mathbf{b}]_{\lambda}^{r}=[\mathbf{b}]_{\lambda+\left(1^{r-1}\right)}^{r-1} \wedge b_{0}\right)$ :

$$
\bar{\sigma}_{-}(w) \sigma_{+}(z)[\mathbf{b}]_{\lambda}^{r}=\left(1-\frac{z}{w}\right) \sigma_{+}(z) \bar{\sigma}_{-}(w)[\mathbf{b}]_{\lambda}^{r} .
$$

Proof As a matter of i), we observe that $\bar{\sigma}_{-}(w) \sigma_{+}(z) b_{\lambda}=\sigma_{+}(z) \bar{\sigma}_{-}(z) b_{\lambda}$ if $\lambda>0$. Indeed 


$$
\begin{array}{rlr}
\bar{\sigma}_{-}(w) \sigma_{+}(z) b_{\lambda} & =\bar{\sigma}_{-}(w)\left(\sum_{j \geq 0} b_{\lambda+j} z^{j}\right) & \\
& =\sum_{j \geq 0}\left(b_{\lambda+j}-\frac{b_{\lambda+j-1}}{w}\right) z^{j} & \text { (Definition of } \left.\sigma_{+}(z) b_{\lambda}\right) \\
& =\sigma_{+}(z) \bar{\sigma}_{-}(w) b_{\lambda} .
\end{array}
$$

Similarly

$$
\bar{\sigma}_{-}(w) \bar{\sigma}_{+}(z) b_{\lambda}=\bar{\sigma}_{+}(z) \bar{\sigma}_{-}(w) b_{\lambda},
$$

as a direct straightforward computation shows. Therefore, under the hypothesis $\ell(\lambda)=r$ :

$$
\begin{aligned}
\bar{\sigma}_{-}(w) \sigma_{+}(z)[\mathbf{b}]_{\lambda}^{r} & =\bar{\sigma}_{-}(w) \sigma_{+}(z) b_{r-1+\lambda_{1}} \wedge \cdots \wedge \bar{\sigma}_{-}(w) \sigma_{+}(z) b_{\lambda_{r}} \\
& =\sigma_{+}(z) \bar{\sigma}_{-}(w) b_{r-1+\lambda_{1}} \wedge \cdots \wedge \sigma_{+}(z) \bar{\sigma}_{-}(w) b_{\lambda_{r}}=\sigma_{+}(z) \bar{\sigma}_{-}(w)[\mathbf{b}]_{\lambda}^{r},
\end{aligned}
$$

and the same can be argued for the commutation of $\bar{\sigma}_{+}(z)$ and $\bar{\sigma}_{-}(w)$.

To prove equality (34), one observes that

$$
\begin{array}{rlr}
\bar{\sigma}_{-}(w) \sigma_{+}(z) b_{0} & =\bar{\sigma}_{-}(w) \sum_{j \geq 0} b_{j} z^{j} & \text { (Def inition of } \left.\sigma_{+}(z) b_{0}\right) \\
& =b_{0}+\sum_{j \geq 1}\left(b_{j}-\frac{b_{j-1}}{w}\right) z^{j} & \text { (Def inition of } \left.\sigma_{-}(w) b_{j}\right) \\
& =b_{0}+\sum_{j \geq 1} b_{j} z^{j}-\frac{z}{w} \sum_{j \geq 0} b_{j} z^{j} & \\
& =\left(1-\frac{z}{w}\right) \sigma_{+}(z) b_{0} \\
& =\left(1-\frac{z}{w}\right) \sigma_{+}(z) \bar{\sigma}_{-}(w) b_{0},
\end{array}
$$

because, in general, $\bar{\sigma}_{-}(w)$ acts on $[\mathbf{b}]_{0}^{r}$ as the identity. So, if $\ell(\lambda)<r$ (i.e. $\lambda_{r}=0$ ) one obtains:

$$
\begin{aligned}
\bar{\sigma}_{-}(w) \sigma_{+}(z)[\mathbf{b}]_{\lambda}^{r} & =\bar{\sigma}_{-}(w) \sigma_{+}(z)\left([\mathbf{b}]_{\lambda+\left(1^{r-1}\right)}^{r-1} \wedge b_{0}\right) & & \text { (Definition of } \left.[\mathbf{b}]_{\lambda}^{r}\right) \\
& =\bar{\sigma}_{-}(w) \sigma_{+}(z)[\mathbf{b}]_{\lambda+\left(1^{r-1}\right)}^{r-1} \wedge \bar{\sigma}_{-}(w) \sigma_{+}(z) b_{0} & & \left(\bar{\sigma}_{-}(w) \sigma_{+}(z)\right. \text { is a HS derivation) } \\
& =\sigma_{+}(z) \bar{\sigma}_{-}(w)[\mathbf{b}]_{\lambda+\left(1^{r-1}\right)}^{r-1} \wedge\left(1-\frac{z}{w}\right) \sigma_{+}(z) \bar{\sigma}_{-}(w) b_{0} & & \text { Commutation (35)) } \\
& =\left(1-\frac{z}{w}\right) \sigma_{+}(z) \bar{\sigma}_{-}(w)[\mathbf{b}]_{\lambda}^{r} . & &
\end{aligned}
$$

\section{Commutation rules for contractions}

The goal of this section is to prove the following 
5.1 Theorem For all $u \in \bigwedge^{r} V$, the following commutation rule holds:

$$
\left.\left.\beta_{0}\right\lrcorner \sigma_{-}(w) \bar{\sigma}_{+}(z) u=\left(1-\frac{z}{w}\right) \bar{\sigma}_{+}(z)\left(\beta_{0}\right\lrcorner \sigma_{-}(w) u\right) .
$$

To prove Theorem 5.1 some preparation is needed.

\subsection{Diagrams for contractions}

Let us begin to introduce a piece of useful notation. If $\beta \in V^{*}$, we represent the contraction

$$
\beta_{\lrcorner}\left(u_{1} \wedge \cdots \wedge u_{r}\right)
$$

as defined by equality (7), via the diagram:

$$
\left|\begin{array}{cccc}
\beta\lrcorner u_{1} & \beta\lrcorner u_{2} & \cdots & \beta_{\lrcorner} u_{r} \\
u_{1} & u_{2} & \cdots & u_{r}
\end{array}\right|=\left|\begin{array}{cccc}
\beta\left(u_{1}\right) & \beta\left(u_{2}\right) & \cdots & \beta\left(u_{r}\right) \\
u_{1} & u_{2} & \ldots & u_{r}
\end{array}\right|,
$$

to be read as follows. The scalar $(-1)^{j+1} \beta_{\lrcorner} u_{j}:=(-1)^{j} \beta\left(u_{j}\right)$ is the coefficient of the element of $\wedge^{r-1} V$ obtained by removing the wedge factor $u_{j}$ from $u_{1} \wedge u_{2} \wedge \cdots \wedge u_{r}$. For example

$$
\left|\begin{array}{ccc}
\beta\lrcorner u_{1} & \beta\lrcorner u_{2} & \beta\lrcorner u_{3} \\
u_{1} & u_{2} & u_{3}
\end{array}\right|=\beta\left(u_{1}\right) \cdot u_{2} \wedge u_{3}-\beta\left(u_{2}\right) u_{1} \wedge u_{3}+\beta\left(u_{3}\right) u_{1} \wedge u_{2} .
$$

This is exactly the expanded expression of the contraction $\beta_{\lrcorner}\left(u_{1} \wedge u_{2} \wedge u_{3}\right)$. Recall now the generating function $\boldsymbol{\beta}\left(w^{-1}\right):=\sum_{j \geq 0} \beta_{j} w^{-j}$ introduced in formula (3).

5.3 Lemma We have:

$$
\left.\boldsymbol{\beta}\left(w^{-1}\right)\right\lrcorner[\mathbf{b}]_{\lambda}^{r}=\left|\begin{array}{cccc}
w^{-r+1-\lambda_{1}} & w^{-r+2-\lambda_{2}} & \cdots & w^{-\lambda_{r}} \\
b_{r-1+\lambda_{1}} & b_{r-2+\lambda_{2}} & \cdots & b_{\lambda_{r}}
\end{array}\right| .
$$

Proof Since $\left.\beta_{j}\right\lrcorner b_{i}=\beta_{j}\left(b_{i}\right)=\delta_{i j}$, it clearly follows that

$$
\left.\boldsymbol{\beta}\left(w^{-1}\right)\right\lrcorner b_{j}=\sum_{i \geq 0} \beta_{i}\left(b_{j}\right) w^{-i}=w^{-j} .
$$

Putting $\boldsymbol{\beta}\left(w^{-1}\right)$ instead of $\beta$ in equality (37), gives (38).

5.4 Proposition For all $u \in \bigwedge^{r} V$ :

$$
\left.\left.\boldsymbol{\beta}\left(w^{-1}\right)\right\lrcorner \bar{\sigma}_{+}(z) u=\left(1-\frac{z}{w}\right) \bar{\sigma}_{+}(z)\left(\boldsymbol{\beta}\left(w^{-1}\right)\right\lrcorner u\right) .
$$

Proof Since each $u \in \bigwedge^{r} V$ is a finite linear combination of $[\mathbf{b}]_{\lambda}^{r}$, it is no harm to assume $u=[\mathbf{b}]_{\lambda}^{r}$. Notice that

$$
\begin{array}{rlrl}
\left.\boldsymbol{\beta}\left(w^{-1}\right)\right\lrcorner \bar{\sigma}_{+}(z) b_{j} & \left.=\boldsymbol{\beta}\left(w^{-1}\right)\right\lrcorner\left(b_{j}-b_{j+1} z\right) & & \left(\text { Def inition of } \bar{\sigma}_{+}(z)\right) \\
& \left.\left.=\boldsymbol{\beta}\left(w^{-1}\right)\right\lrcorner b_{j}-\boldsymbol{\beta}\left(w^{-1}\right)\right\lrcorner b_{j+1} z & & \left.\left(\text { Action of } \boldsymbol{\beta}\left(w^{-1}\right)\right\lrcorner\right) \\
& =\frac{1}{w^{j}}-\frac{z}{w^{j+1}}=\frac{1}{w^{j}}\left(1-\frac{z}{w}\right) . &
\end{array}
$$


By expressing the contraction via diagram (37), one has:

$$
\begin{aligned}
& \left.\boldsymbol{\beta}\left(w^{-1}\right)\right\lrcorner \bar{\sigma}_{+}(z)[\mathbf{b}]_{\lambda}^{r} \\
& =\left|\begin{array}{cccc}
\left.\boldsymbol{\beta}\left(w^{-1}\right)\right\lrcorner \bar{\sigma}_{+}(z) b_{r-1+\lambda_{1}} & \left.\boldsymbol{\beta}\left(w^{-1}\right)\right\lrcorner \bar{\sigma}_{+}(z) b_{r-2+\lambda_{2}} & \cdots & \left.\boldsymbol{\beta}\left(w^{-1}\right)\right\lrcorner \bar{\sigma}_{+}(z) b_{\lambda_{r}} \\
\bar{\sigma}_{+}(z) b_{r-1+\lambda_{1}} & \bar{\sigma}_{+}(z) b_{r-2+\lambda_{2}} & \cdots & \bar{\sigma}_{+}(z) b_{\lambda_{r}}
\end{array}\right|,
\end{aligned}
$$

which by (40) is equal to:

$$
\begin{aligned}
& =\left|\begin{array}{cccc}
\left(1-\frac{z}{w}\right) \frac{1}{w^{r-1+\lambda_{1}}} & \left(1-\frac{z}{w}\right) \frac{1}{w^{r-2+\lambda_{2}}} & \cdots & \left(1-\frac{z}{w}\right) \frac{1}{w^{\lambda_{r}}} \\
\bar{\sigma}_{+}(z) b_{r-1+\lambda_{1}} & \bar{\sigma}_{+}(z) b_{r-2+\lambda_{2}} & \cdots & \bar{\sigma}_{+}(z) b_{\lambda_{r}}
\end{array}\right| \\
& =\left(1-\frac{z}{w}\right)\left|\begin{array}{cccc}
\frac{1}{w^{r-1+\lambda_{1}}} & \frac{1}{w^{r-2+\lambda_{2}}} & \cdots & \frac{1}{w^{\lambda_{r}}} \\
\bar{\sigma}_{+}(z) b_{r-1+\lambda_{1}} & \bar{\sigma}_{+}(z) b_{r-2+\lambda_{2}} & \cdots & \bar{\sigma}_{+}(z) b_{\lambda_{r}}
\end{array}\right| .
\end{aligned}
$$

Since the determinant occurring in (41) is a linear combination of $\left[\bar{\sigma}_{+}(z) \mathbf{b}\right]_{\lambda^{(j)}}^{r-1}=\bar{\sigma}_{+}(z)[\mathbf{b}]_{\lambda^{(j)}}^{r-1}$ (because $\bar{\sigma}_{+}(z)$ is a HS derivation), where we denoted by $\lambda^{(j)}$ the partition of lenght at most $r-1$ obtained by omitting the $j$-th part, it follows that the action of $\bar{\sigma}_{+}(z)$ can be factorized from the bottom row of (41), giving

$$
\left.\left(1-\frac{z}{w}\right) \bar{\sigma}_{+}(z)\left|\begin{array}{llll}
\frac{1}{w^{r-1+\lambda_{1}}} & \frac{1}{w^{r-2+\lambda_{2}}} & \cdots & \frac{1}{w^{\lambda_{r}}} \\
b_{r-1+\lambda_{1}} & b_{r-2+\lambda_{2}} & \cdots & b_{\lambda_{r}}
\end{array}\right|=\left(1-\frac{z}{w}\right) \bar{\sigma}_{+}(z)\left(\boldsymbol{\beta}\left(w^{-1}\right)\right\lrcorner[\mathbf{b}]_{\lambda}^{r}\right),
$$

which ends the proof of the Proposition.

5.5 Lemma For all $u \in \bigwedge^{r} V$,

$$
\left.\left.\boldsymbol{\beta}\left(w^{-1}\right)\right\lrcorner u=\bar{\sigma}_{-}(w)\left(\beta_{0}\right\lrcorner \sigma_{-}(w) u\right) .
$$

Proof It is basically contained in [19, Proposition 4.3] but, because some mild difformity in the notation, we prefer to repeat it here. Recall the definition of transpose of a HS derivation on $\bigwedge V$. We observe that $\boldsymbol{\beta}\left(w^{-1}\right)=\sigma_{-}(w)^{T} \beta_{0}$. Then, for all $\eta \in \bigwedge^{r-1} V^{*}$,

$$
\begin{aligned}
& \left.\eta\left(\beta\left(w^{-1}\right)\right\lrcorner u\right)=\left(\beta\left(w^{-1}\right) \wedge \eta\right)(u) \quad \text { (Def inition } 2.2 \text { of contraction) } \\
& =\left(\sigma_{-}(w)^{T} \beta_{0} \wedge \eta\right)(u) \quad \text { (by the above observation) } \\
& =\sigma_{-}(w)^{T}\left(\beta_{0} \wedge \bar{\sigma}_{-}(w)^{T} \eta\right)(u) \quad \text { (Integration by parts) } \\
& \left.=\beta_{0} \wedge \bar{\sigma}_{-}(w)^{T} \eta\left(\sigma_{-}(w) u\right) \quad \text { (Definition of transpose of } \bar{\sigma}_{-}(w)^{T}\right) \\
& =\bar{\sigma}_{-}(w)^{T} \eta\left(\beta_{0} \sigma_{-}(w) u\right) \quad \text { (Again 2.2) } \\
& \left.=\eta\left(\bar{\sigma}_{-}(w)\left(\beta_{0}\right\lrcorner \sigma_{-}(w) u\right)\right) \quad \text { (Def inition of transpose). }
\end{aligned}
$$


The last equality proves (42), due to the arbitrary choice of $\eta \in \bigwedge^{r-1} V^{*} \cong\left(\bigwedge^{r-1} V\right)^{*}$.

5.6 Lemma The operator $\bar{\sigma}_{+}(z)$ commutes with contracting against $\beta_{0}$, i.e. for all $\lambda \in \mathcal{P}_{r}$

$$
\left.\left.\beta_{0}\right\lrcorner \bar{\sigma}_{+}(z)[\mathbf{b}]_{\lambda}^{r}=\bar{\sigma}_{+}(z)\left(\beta_{0}\right\lrcorner[\mathbf{b}]_{\lambda}^{r}\right) .
$$

Proof There are two cases. If $\ell(\lambda)=r$, both members of (43) vanish. If $\ell(\lambda) \leq r-1$, then $\left.\left.\beta_{0}\right\lrcorner \bar{\sigma}_{+}(z)[\mathbf{b}]_{\lambda}^{r}=(-1)^{r-1} \bar{\sigma}_{+}(z)[\mathbf{b}]_{\lambda+\left(1^{(r-1)}\right)}^{r-1}=\bar{\sigma}_{+}(z)\left(\beta_{0}\right\lrcorner[\mathbf{b}]_{\lambda}^{r}\right)$.

We are now in position to provide the

\subsection{Proof of Theorem 5.1}

We have:

$$
\begin{array}{rlrl}
\left.\beta_{0}\right\lrcorner \sigma_{-}(w) \bar{\sigma}_{+}(z) u & \left.=\sigma_{-}(w) \bar{\sigma}_{-}(w)\left(\beta_{0}\right\lrcorner \sigma_{-}(w) \bar{\sigma}_{+}(z) u\right)\left(\sigma_{-}(w) \bar{\sigma}_{-}(w)=1\right) & \\
& \left.=\sigma_{-}(w)\left(\beta\left(w^{-1}\right)\right\lrcorner \bar{\sigma}_{+}(z) u\right) & & (\text { Lemma 5.5) } \\
& \left.=\left(1-\frac{z}{w}\right) \sigma_{-}(w) \bar{\sigma}_{+}(z)\left(\beta\left(w^{-1}\right)\right\lrcorner u\right) & & \text { (Proposition 5.4) } \\
& \left.=\left(1-\frac{z}{w}\right) \sigma_{-}(w) \bar{\sigma}_{+}(z) \bar{\sigma}_{-}(w)\left(\beta_{0}\right\lrcorner \sigma_{-}(w) u\right) & & \text { (again Lemma 5.5) } \\
& \left.=\left(1-\frac{z}{w}\right) \sigma_{-}(w) \bar{\sigma}_{-}(w) \bar{\sigma}_{+}(z)\left(\beta_{0}\right\lrcorner \sigma_{-}(w) u\right) & & (\text { formula }(33) \text { of Proposition 4.4) } \\
& \left.=\left(1-\frac{z}{w}\right) \bar{\sigma}_{+}(z)\left(\beta_{0}\right\lrcorner \sigma_{-}(w) u\right) & & \left(\sigma_{-}(w) \bar{\sigma}_{-}(w)=1\right) .
\end{array}
$$

and Theorem 5.1 is thence proven.

\section{The vertex operator $\Gamma\left(z_{k}\right)$}

The main purpose of this section is to interpret the vertex operator $\Gamma\left(\mathbf{z}_{k}\right)$, introduced in Definition 4.3, formula (30), in terms of wedging operation on the exterior algebra. It will generalise [19, Proposition 4.2]. This will be achieved in Theorem 6.5 below and will be used in our Main Theorem 8.5.

6.1 Lemma For all $j \geq 0$ and all $k \geq 1$ one has

$$
\bar{\sigma}_{+}\left(\mathbf{z}_{k}\right) b_{j}=b_{j}+\sum_{i=1}^{k}(-1)^{i} e_{i}\left(\mathbf{z}_{k}\right) b_{j+i}
$$

and

$$
\sigma_{+}\left(\mathbf{z}_{k}\right) b_{j}=b_{j}+\sum_{i \geq 1}^{k} h_{i}\left(\mathbf{z}_{k}\right) b_{j+i},
$$

where $e_{i}\left(\mathbf{z}_{k}\right)$ and $h_{i}\left(\mathbf{z}_{k}\right)$ are, respectively, the elementary and complete symmetric polynomial of degree $i$ in the indeterminates $\mathbf{z}_{k}:=\left(z_{1}, \ldots, z_{k}\right)$. 
Proof Formula (44) is the content of [21, Lemma 5.7] to which we refer to. Formula (45) is a consequence of (44), keeping into account that $\sigma_{+}\left(\mathbf{z}_{k}\right)$ and $\bar{\sigma}_{+}\left(\mathbf{z}_{k}\right)$ are mutually inverse in $\operatorname{End}_{\mathbb{Q}}(\bigwedge V)\left[\mathbf{z}_{k}\right]$.

6.2 Lemma One has:

$$
\bar{\sigma}_{-}\left(\mathbf{z}_{k}\right) b_{j+k}=b_{j+k}+\sum_{i=1}^{k}(-1)^{i} e_{i}\left(\mathbf{z}_{k}^{-1}\right) b_{j+k-i},
$$

where $e_{i}\left(\mathbf{z}_{k}^{-1}\right)=e_{i}\left(z_{1}^{-1}, \ldots, z_{k}^{-1}\right)$ is the elementary symmetric polynomial of degree $i$ in $\left(z_{1}^{-1}, \ldots, z_{k}^{-1}\right)$.

Proof The proof works the same as in [21, Lemma 6.7]. The formula is true for $k=1$, because

$$
\bar{\sigma}_{-}\left(z_{1}\right) b_{j+1}=b_{j+1}-\frac{b_{j}}{z_{1}} .
$$

By induction, suppose that (46) holds for $k-1 \geq 0$. Then it holds for $k$. Indeed

$$
\begin{aligned}
& \bar{\sigma}_{-}\left(z_{1}\right) \bar{\sigma}_{-}\left(z_{2}\right) \ldots \bar{\sigma}_{-}\left(z_{k}\right) b_{j+k} \\
& =\bar{\sigma}_{-}\left(z_{1}\right)\left[b_{j+k}-e_{1}\left(\frac{1}{z_{2}}, \cdots, \frac{1}{z_{k}}\right) b_{j+k-1}+\cdots+(-1)^{k-1} e_{k-1}\left(\frac{1}{z_{2}}, \cdots, \frac{1}{z_{k}}\right) b_{j+1}\right] \\
& =b_{j+k}-\frac{b_{j+k-1}}{z_{1}}-e_{1}\left(\frac{1}{z_{2}}, \cdots, \frac{1}{z_{k}}\right)\left(b_{j+k-1}-\frac{b_{j+k-2}}{z_{1}}\right)+\cdots \\
& \quad+(-1)^{k-1} e_{k-1}\left(\frac{1}{z_{2}}, \cdots, \frac{1}{z_{k}}\right)\left(b_{j+1}-\frac{b_{j}}{z_{1}}\right) \\
& =b_{j+k}+\sum_{i=1}^{k}(-1)^{i} e_{i}\left(\frac{1}{z_{1}}, \cdots, \frac{1}{z_{k}}\right) b_{j+k-i},
\end{aligned}
$$

as desired.

6.3 Lemma The following equality holds for all $1 \leq i \leq k$ :

$$
\frac{e_{i}\left(\mathbf{z}_{k}\right)}{z_{1} \cdots z_{k}}=e_{k-i}\left(\frac{1}{z_{1}}, \ldots, \frac{1}{z_{k}}\right) .
$$

Proof Recall the following definition of the elementary symmetric polynomials in $k$ indeterminates through generating functions:

$$
\sum_{i=0}^{k} e_{i}\left(\mathbf{z}_{k}\right) t^{i}=\prod_{i=1}^{k}\left(1+z_{i} t\right)
$$

By dividing both sides of (48) by $e_{k}\left(\mathbf{z}_{k}\right)=z_{1} \ldots z_{k}$ we get

$$
\sum_{i=0}^{k} \frac{e_{i}\left(\mathbf{z}_{k}\right)}{z_{1} \cdots z_{k}} t^{i}=\prod_{i=1}^{k}\left(\frac{1}{z_{i}}+t\right) .
$$


The claim then follows by comparing the coefficient of $t^{i}$ in in either side of (49).

6.4 Lemma For all $k \geq 1, r \geq 0$ and $\lambda \in \mathcal{P}_{r}$ :

$$
[\mathbf{b}]_{0}^{k} \wedge \bar{\sigma}_{+}\left(\mathbf{z}_{k}\right)[\mathbf{b}]_{\lambda}^{r}=e_{k}\left(\mathbf{z}_{k}\right)^{r} \bar{\sigma}_{-}\left(\mathbf{z}_{k}\right)[\mathbf{b}]_{\lambda}^{r+k} .
$$

Proof Equality (50) holds for $r=1$ :

$$
\begin{aligned}
& {[\mathbf{b}]_{0}^{k} \wedge \bar{\sigma}_{+}\left(\mathbf{z}_{k}\right)[\mathbf{b}]_{\lambda}^{1}=[\mathbf{b}]_{0}^{k} \wedge \bar{\sigma}_{+}\left(\mathbf{z}_{k}\right) b_{\lambda}} \\
& =[\mathbf{b}]_{0}^{k} \wedge\left(b_{\lambda}-e_{1}\left(\mathbf{z}_{k}\right) b_{\lambda+1}+\cdots+(-1)^{k} e_{k}\left(\mathbf{z}_{k}\right) b_{\lambda+k}\right) \\
& =[\mathbf{b}]_{0}^{k} \wedge(-1)^{k} e_{k}\left(\mathbf{z}_{k}\right)\left[b_{\lambda+k}-\frac{e_{k-1}\left(\mathbf{z}_{k}\right)}{e_{k}\left(\mathbf{z}_{k}\right)} b_{\lambda+k-1}+\cdots+(-1)^{k} \frac{1}{e_{k}\left(\mathbf{z}_{k}\right)} b_{\lambda}\right] \\
& =e_{k}\left(\mathbf{z}_{k}\right) \bar{\sigma}_{-}\left(\mathbf{z}_{k}\right) b_{\lambda+k} \wedge[\mathbf{b}]_{0}^{k} \\
& =e_{k}\left(\mathbf{z}_{k}\right) \bar{\sigma}_{-}\left(\mathbf{z}_{k}\right)\left(b_{\lambda+k} \wedge \bar{\sigma}_{-}\left(\mathbf{z}_{k}\right)[\mathbf{b}]_{0}^{k}\right) \\
& =e_{k}\left(\mathbf{z}_{k}\right) \bar{\sigma}_{-}\left(\mathbf{z}_{k}\right)\left(b_{\lambda+k} \wedge b_{k-1} \wedge \ldots \wedge b_{0}\right) \\
& =e_{k}\left(\mathbf{z}_{k}\right) \bar{\sigma}_{-}\left(\mathbf{z}_{k}\right)[\mathbf{b}]_{\lambda}^{1+k} .
\end{aligned}
$$

(By Factorization)$$
\left(\bar{\sigma}_{-}\left(\mathbf{z}_{k}\right)[\mathbf{b}]_{0}^{k}=[\mathbf{b}]_{0}^{k}\right)
$$

Therefore the property is true for $r=1$. Assume now (50) holds true for $r-1 \geq 0$. Then

$$
\begin{aligned}
{[\mathbf{b}]_{0}^{k} \wedge \bar{\sigma}_{+}\left(\mathbf{z}_{k}\right)[\mathbf{b}]_{\lambda}^{r} } & =[\mathbf{b}]_{0}^{k} \wedge \bar{\sigma}_{+}\left(\mathbf{z}_{k}\right) b_{r-1+\lambda_{1}} \wedge \ldots \wedge \bar{\sigma}_{+}\left(\mathbf{z}_{k}\right) b_{\lambda_{r}} \\
& =[\mathbf{b}]_{0}^{k} \wedge(-1)^{k} e_{k}\left(\mathbf{z}_{k}\right) \bar{\sigma}_{-}\left(\mathbf{z}_{k}\right) b_{r-1+k+\lambda_{1}} \wedge \ldots \wedge(-1)^{k} e_{k}\left(\mathbf{z}_{k}\right) \bar{\sigma}_{-}\left(\mathbf{z}_{k}\right) b_{\lambda_{r}+k} \\
& =e_{k}\left(\mathbf{z}_{k}\right)^{r}\left[\bar{\sigma}_{-}\left(\mathbf{z}_{k}\right) b_{r+k-1+\lambda_{1}} \wedge \ldots \wedge \bar{\sigma}_{-}\left(\mathbf{z}_{k}\right) b_{k+\lambda_{r}} \wedge \bar{\sigma}_{-}\left(\mathbf{z}_{k}\right)[\mathbf{b}]_{0}^{k}\right] \\
& =e_{k}\left(\mathbf{z}_{k}\right)^{r} \bar{\sigma}_{-}\left(\mathbf{z}_{k}\right)\left(b_{r+k-1+\lambda_{1}} \wedge b_{k+\lambda_{r}} \wedge b_{k} \wedge \ldots \wedge b_{0}\right) \\
& =e_{k}\left(\mathbf{z}_{k}\right)^{r} \bar{\sigma}_{-}\left(\mathbf{z}_{k}\right)[\mathbf{b}]_{\lambda}^{r+k},
\end{aligned}
$$

as claimed.

6.5 Theorem For all $u \in \bigwedge^{r} V\left[\mathbf{w}_{k}, \mathbf{w}_{k}^{-1}\right]$ we have:

$$
\sigma_{+}\left(z_{1}, \ldots, z_{k}\right)[\mathbf{b}]_{0}^{k} \wedge u=\prod_{j=1}^{r} z_{j}^{r} \cdot \Gamma\left(\mathbf{z}_{k}\right) u,
$$

the equality holding in $\bigwedge^{r+k} V \llbracket \mathbf{z}_{k}, \mathbf{w}_{k} \rrbracket\left[\mathbf{w}_{k}^{-1}\right]$.

Proof Recall that we consider all the Schubert derivations extended by linearity over rings of formal power series with rational coefficients. See definition 2.5. Then our arbitrary $u$ is intended as a linear combination of $[\mathbf{b}]_{\lambda}^{r}$ with coefficients being polynomials. Then we can assume with no harm that $u=[\mathbf{b}]_{\lambda}^{r}$, a basis element of $\bigwedge^{r} V$. Keeping the same notation as in Sect. 2, we first apply integration by parts. Then

$$
\begin{aligned}
\sigma_{+}\left(\mathbf{z}_{k}\right)[\mathbf{b}]_{0}^{k} \wedge[\mathbf{b}]_{\lambda}^{r} & =\sigma_{+}\left(\mathbf{z}_{k}\right)\left([\mathbf{b}]_{0}^{k} \wedge \bar{\sigma}_{+}\left(\mathbf{z}_{k}\right)[\mathbf{b}]_{\lambda}^{r}\right) & & \text { (By integration by parts (14)) } \\
& =\sigma_{+}\left(\mathbf{z}_{k}\right) e_{k}\left(\mathbf{z}_{k}\right)^{r} \bar{\sigma}_{-}\left(\mathbf{z}_{k}\right)[\mathbf{b}]_{\lambda}^{r+k} & & (\text { By Lemma 6.4) } \\
& =e_{k}\left(\mathbf{z}_{k}\right)^{r} \sigma_{+}\left(\mathbf{z}_{k}\right) \bar{\sigma}_{-}\left(\mathbf{z}_{k}\right)[\mathbf{b}]_{\lambda}^{r+k} & & \\
& =\prod_{j=1}^{k} z_{j}^{r} \Gamma\left(\mathbf{z}_{k}\right)[\mathbf{b}]_{\lambda}^{r} & & \text { (Def inition of } \left.\Gamma\left(\mathbf{z}_{k}\right)\right)
\end{aligned}
$$


as desired.

If $k=1$, and $z=z_{1}$, one obtains

$$
\sigma_{+}(z) b_{0} \wedge[\mathbf{b}]_{\lambda}^{r}=z^{r} \Gamma(z)[\mathbf{b}]_{\lambda}^{r}=z^{r} \sigma_{+}(z) \bar{\sigma}_{-}(z)[\mathbf{b}]_{\lambda}^{r+1},
$$

which is precisely [19, Proposition 5.4] or [20, Proposition 3.2]. Their shape there looks more involved because of a different notation.

\section{The vertex operator $\Gamma^{*}\left(\mathbf{w}_{k}\right)$}

In the same vein of Sect. 6, the present one will be devoted to interpret the action of the vertex operator $\Gamma^{*}\left(\mathbf{w}_{k}\right)$ on $\bigwedge V$ in terms of contraction operators. The output will be Theorem 7.3, stated at the end of the section, another building block of the main Theorem 8.5. We begin with some preparation.

7.1 Lemma The following equality holds for all $r \geq 1$ and all $\lambda \in \mathcal{P}_{r}$ :

$$
\bar{\sigma}_{-r+1}\left(\beta_{0\lrcorner} \sigma_{-}(w)[\mathbf{b}]_{\lambda}^{r}\right)=\Delta_{\lambda}\left(\sigma_{-}(w) H_{r-1}\right)[\mathbf{b}]_{0}^{r-1} .
$$

Proof This is [19, Lemma 5.8].

7.2 Lemma

$$
\left.\boldsymbol{\beta}\left(w^{-1}\right)\right\lrcorner[\mathbf{b}]_{\lambda}^{r}=w^{-r+1} \bar{\sigma}_{+}(w) \Delta_{\lambda}\left(\sigma_{-}(w) H_{r-1}\right)[\mathbf{b}]_{0}^{r-1} .
$$

Proof Invoking Lemma 5.5,

$$
\left.\left.\boldsymbol{\beta}\left(w^{-1}\right)\right\lrcorner[\mathbf{b}]_{\lambda}^{r}=\bar{\sigma}_{-}(w)\left(\beta_{0}\right\lrcorner \sigma_{-}(w)[\mathbf{b}]_{\lambda}^{r}\right) .
$$

Since $\left.\beta_{0}\right\lrcorner \sigma_{-}(w)[\mathbf{b}]_{\lambda}^{r}$ is a linear combination of $[\mathbf{b}]_{\mu}^{r-1}$ with $\ell(\boldsymbol{\mu})=r-1$ (i.e. no $b_{0}$ occurs in the monomial), then by [19, Proposition 4.3]

$$
\left.\left.\bar{\sigma}_{-}(w)\left(\beta_{0}\right\lrcorner \sigma_{-}(w)[\mathbf{b}]_{\lambda}^{r}\right)=w^{-r+1} \bar{\sigma}_{+}(w) \bar{\sigma}_{-r+1}\left(\beta_{0}\right\lrcorner \sigma_{-}(w)[\mathbf{b}]_{\lambda}^{r}\right) .
$$

Using Lemma 7.1 one obtains (53).

7.3 Theorem The following equality holds:

$$
\left(\boldsymbol{\beta}\left(w_{k}^{-1}\right) \wedge \boldsymbol{\beta}\left(w_{k-1}^{-1}\right) \wedge \cdots \wedge \boldsymbol{\beta}\left(w_{1}^{-1}\right)\right)_{\lrcorner}[\mathbf{b}]_{\lambda}^{r}=\frac{\Delta_{0}\left(\mathbf{w}_{k}\right)}{\left(w_{1} \cdots w_{k}\right)^{r-1}} \Gamma^{*}\left(\mathbf{w}_{k}\right)[\mathbf{b}]_{\lambda}^{r} .
$$

where $\Delta_{0}\left(\mathbf{w}_{k}\right)$ denotes the Vandermonde determinant $\prod_{1 \leq i<j \leq k}\left(w_{j}-w_{i}\right)$.

Proof For $k=1$ the property

$$
\left.\boldsymbol{\beta}\left(w_{1}^{-1}\right)\right\lrcorner[\mathbf{b}]_{\lambda}^{r}=w_{1}^{-r+1} \bar{\sigma}_{+}\left(w_{1}\right) \Delta_{\lambda}\left(\sigma_{-}\left(w_{1}\right) H_{r-1}\right)[\mathbf{b}]_{0}^{r-1}
$$

is just Lemma 7.2. Arguing by induction, let us assume the claim holding true for $0 \leq k-1 \leq r-1$ and let us show it holds for $k$. We have

$$
\left.\left.\left.\boldsymbol{\beta}\left(w_{k}^{-1}\right) \wedge \boldsymbol{\beta}\left(w_{k-1}^{-1}\right) \wedge \cdots \wedge \boldsymbol{\beta}\left(w_{1}^{-1}\right)\right\lrcorner[\mathbf{b}]_{\lambda}^{r}=\boldsymbol{\beta}\left(w_{k}^{-1}\right)\right\lrcorner\left(\boldsymbol{\beta}\left(w_{k-1}^{-1}\right) \wedge \cdots \wedge \boldsymbol{\beta}\left(w_{1}^{-1}\right)\right\lrcorner[\mathbf{b}]_{\lambda}^{r}\right) .
$$


Using the inductive hypothesis:

$$
\left.=\boldsymbol{\beta}\left(w_{k}^{-1}\right)\right\lrcorner \frac{\Delta_{0}\left(\mathbf{w}_{k-1}\right)}{\left(w_{1} \cdots w_{k-1}\right)^{r-1}} \bar{\sigma}_{+}\left(\mathbf{w}_{k-1}\right) \Delta_{\lambda}\left(\sigma_{-}\left(\mathbf{w}_{k-1}\right) H_{r-k+1}\right)[\mathbf{b}]_{0}^{r-k+1} .
$$

By applying Lemma 7.2, one gets:

$$
\begin{aligned}
& \left.=w_{k}^{r-k+1} \bar{\sigma}_{+}\left(w_{k}\right)\left[\beta_{0}\right\lrcorner \sigma_{-}\left(w_{k}\right) \bar{\sigma}_{+}\left(\mathbf{w}_{k-1}\right) \Delta_{\lambda}\left(\sigma_{-}\left(\mathbf{w}_{k-1}\right) H_{r-k+1}\right)[\mathbf{b}]_{0}^{r-k+1}\right] \\
& \cdot \frac{\Delta_{0}\left(\mathbf{w}_{k-1}\right)}{\left(w_{1} \cdots w_{k-1}\right)^{r-1}}
\end{aligned}
$$

Now we use the commutation rules prescribed by Theorem 5.1 and Lemma 5.6:

$$
\begin{aligned}
= & w_{k}^{k-r} \bar{\sigma}_{+}\left(w_{k}\right)\left[\prod_{j=0}^{k-1}\left(1-\frac{w_{j}}{w_{k}}\right) \cdot\left(\beta_{0} \bar{\sigma}_{+}\left(\mathbf{w}_{k-1}\right) \sigma_{-}\left(\mathbf{w}_{k}\right) \Delta_{\lambda}\left(\sigma_{-}\left(\mathbf{w}_{k-1}\right) H_{r-k+1}\right)[\mathbf{b}]_{0}^{r-k+1}\right)\right] \\
& \cdot \frac{\Delta_{0}\left(\mathbf{w}_{k-1}\right)}{\left(w_{1} \cdots w_{k-1}\right)^{r-1}} \\
= & \frac{w_{k}^{k-r} \Delta_{0}\left(\mathbf{w}_{k-1}\right)}{\left(w_{1} \cdots w_{k-1}\right)^{r-1}} \prod_{j=0}^{k-1}\left(1-\frac{w_{j}}{w_{k}}\right) . \\
& \cdot \bar{\sigma}_{+}\left(w_{k}\right) \bar{\sigma}_{+}\left(\mathbf{w}_{k-1}\right)\left[\left(\beta_{0} \sigma_{-}\left(\mathbf{w}_{k}\right) \Delta_{\lambda}\left(\sigma_{-}\left(\mathbf{w}_{k-1}\right) H_{r-k+1}\right)[\mathbf{b}]_{0}^{r-k+1}\right)\right] \\
= & \frac{\Delta_{0}\left(\mathbf{w}_{k-1}\right) \prod_{j=1}^{k-1}\left(w_{k}-w_{j}\right)}{w_{k}^{r-k} \cdot w_{k}^{k-1}\left(w_{1} \cdots w_{k-1}\right)^{r-1}} \bar{\sigma}_{+}\left(\mathbf{w}_{k}\right) \Delta_{\lambda}\left(\sigma_{-}\left(w_{k}\right) \sigma_{-}\left(\mathbf{w}_{k-1}\right) H_{r-k}\right)[\mathbf{b}]_{0}^{r-k} \\
= & \frac{\Delta_{0}\left(\mathbf{w}_{k}\right)}{\left(w_{1} \cdots w_{k-1} \cdot w_{k}\right)^{r-1}} \cdot \bar{\sigma}_{+}\left(\mathbf{w}_{k}\right) \Delta_{\lambda}\left(\sigma_{-}\left(\mathbf{w}_{k}\right) H_{r-k}\right)[\mathbf{b}]_{0}^{r-k} \\
= & \frac{\Delta_{0}\left(\mathbf{w}_{k}\right)}{\left(w_{1} \cdots w_{k-1} \cdot w_{k}\right)^{r-1}} \Gamma^{*}\left(\mathbf{w}_{k}\right)[\mathbf{b}]_{\lambda}^{r},
\end{aligned}
$$

as claimed.

\section{The main theorem and its declinations}

In this section we shall be concerned with the several declinations of the main theorem describing the $B_{r}$ representation of $g l\left(\bigwedge^{k} V\right)$.

\subsection{Preparation}

Let $g l\left(\bigwedge^{k} V\right):=\bigwedge^{k} V \otimes \bigwedge^{k} V^{*}$. It is a Lie sub-algebra of the endomorphisms of $\bigwedge^{k} V$, with respect to the natural commutator. With the same notation as in 1.1, a basis of $\bigwedge^{k} V \otimes \bigwedge^{k} V^{*}$ is $\left([\mathbf{b}]_{\mu}^{k} \otimes[\boldsymbol{\beta}]_{v}^{k}\right)_{\boldsymbol{\mu}, \boldsymbol{v} \in \mathcal{P}_{k}}$, i.e.

$$
\bigwedge^{k} V \otimes \bigwedge^{k} V^{*}=\bigoplus_{\mu, v \in \mathcal{P}_{k}} \mathbb{Q} \cdot[\mathbf{b}]_{\mu}^{k} \otimes[\boldsymbol{\beta}]_{\nu}^{k},
$$


where $[\boldsymbol{\beta}]_{\nu}^{k}\left([\mathbf{b}]_{\mu}^{k}\right)=\delta_{\mu, \nu}$.

Then the $g l\left(\bigwedge^{k} V\right)$-module structure of $B_{r}$ is defined through the following equality holding in $\bigwedge^{r} V$ :

$$
\left.\left([\mathbf{b}]_{\mu}^{k} \otimes[\boldsymbol{\beta}]_{v}^{k} \star \Delta_{\lambda}\left(H_{r}\right)\right)[\mathbf{b}]_{0}^{r}=[\mathbf{b}]_{\mu}^{k} \wedge\left([\boldsymbol{\beta}]_{\nu}^{k}\right\lrcorner[\mathbf{b}]_{\lambda}^{r}\right) .
$$

This action is very easy to describe in the case $k=r$, but it becomes trickier when $r-k>0$. To describe it we shall consider the generating function

$$
\mathcal{E}\left(\mathbf{z}_{k}, \mathbf{w}_{k}^{-1}\right):=\sum_{\mu, v}[\mathbf{b}]_{\mu}^{k} \otimes[\boldsymbol{\beta}]_{v}^{k} \cdot s_{\mu}\left(\mathbf{z}_{k}\right) s_{v}\left(\mathbf{w}_{k}^{-1}\right): B_{r} \rightarrow B_{r}\left[\left[\mathbf{z}_{k}, \mathbf{w}_{k}\right]\right]\left[\mathbf{z}_{k}^{-1}, \mathbf{w}_{k}^{-1}\right] .
$$

Our main result will consist in the explicit description of $\mathcal{E}\left(\mathbf{z}_{k}, \mathbf{w}_{k}^{-1}\right) \Delta_{\lambda}\left(H_{r}\right)$ in case $k \leq r$ (because otherwise one would obtain the trivial null action), where $\mathcal{E}\left(\mathbf{z}_{k}, \mathbf{w}_{k}^{-1}\right) \Delta_{\lambda}\left(H_{r}\right)$ is such that

$$
\left.\left(\mathcal{E}\left(\mathbf{z}_{k}, \mathbf{w}_{k}^{-1}\right) \Delta_{\lambda}\left(H_{r}\right)\right)[\mathbf{b}]_{0}^{r}=\sum_{\mu \in \mathcal{P}_{k}} s_{\mu}\left(\mathbf{z}_{k}\right)[\mathbf{b}]_{\mu}^{k} \wedge\left(s_{\nu}\left(\mathbf{w}_{k}^{-1}\right)[\boldsymbol{\beta}]_{\nu}^{k}\right\lrcorner[\mathbf{b}]_{\lambda}^{r}\right)
$$

and where $s_{\mu}\left(\mathbf{z}_{k}\right)$ and $s_{v}\left(\mathbf{w}_{k}^{-1}\right)$ denote the Schur symmetric polynomials labeled by the partitions related to the variables $\mathbf{z}_{k}$ and $\mathbf{w}_{k}^{-1}$ respectively.

8.2 Lemma The generating function of the basis $\left([\mathbf{b}]_{\mu}^{k}\right)_{\boldsymbol{\mu} \in \mathcal{P}_{k}}$ of $\bigwedge^{k} V$ is:

$$
\sum_{\boldsymbol{\mu} \in \mathcal{P}_{k}} s_{\boldsymbol{\mu}}\left(\mathbf{z}_{k}\right)[\mathbf{b}]_{\mu}^{k}=\sigma_{+}\left(\mathbf{z}_{k}\right)[\mathbf{b}]_{0}^{k} .
$$

Proof By exploiting the definition of the $B_{k}$-module structure of $\bigwedge^{k} V$, we have

$$
\begin{aligned}
\sum_{\mu \in \mathcal{P}_{k}} s_{\mu}\left(\mathbf{z}_{k}\right)[\mathbf{b}]_{\mu}^{k} & \left.=\sum_{\mu \in \mathcal{P}_{k}} s_{\mu}\left(\mathbf{z}_{k}\right) \Delta_{\mu}\left(H_{k}\right)[\mathbf{b}]_{0}^{k} \quad \text { (using the } \mathrm{B}_{\mathrm{k}}-\text { module structure of } \bigwedge^{k} V\right) \\
& =\left(\sum_{\mu \in \mathcal{P}_{k}} s_{\mu}\left(\mathbf{z}_{k}\right) \Delta_{\mu}\left(H_{k}\right)\right)[\mathbf{b}]_{0}^{k} \\
& =\prod_{j=1}^{k}\left(1+h_{1} z_{j}+h_{2} z_{j}^{2}+\cdots\right)[\mathbf{b}]_{0}^{k} \\
& =\frac{1}{E_{k}\left(z_{1}\right)} \cdot \frac{1}{E_{k}\left(z_{2}\right)} \cdots \frac{1}{E_{k}\left(z_{k}\right)}[\mathbf{b}]_{0}^{k} \quad \text { (By Cauchy formula as in [12, Proposition 2, (iii)]) } \\
& =\sigma_{+}\left(\mathbf{z}_{k}\right)[\mathbf{b}]_{0}^{k} .
\end{aligned}
$$

8.3 Lemma The generating function of the basis elements $\bigwedge^{k} V^{*}$ is:

$$
\sum_{\nu \in \mathcal{P}_{k}} s_{\nu}\left(\mathbf{w}_{k}^{-1}\right)[\boldsymbol{\beta}]_{v}^{k}=\frac{\prod_{j=1}^{k} w_{j}^{k-1}}{\Delta_{0}\left(\mathbf{w}_{k}\right)} \cdot \boldsymbol{\beta}\left(w_{k}^{-1}\right) \wedge \cdots \wedge \boldsymbol{\beta}\left(w_{1}^{-1}\right) .
$$

Proof The one we propose consists in expanding the wedge product of the generating series of the basis $\left(\beta_{j}\right)_{j \geq 0}$ of $V^{*}$ : 


$$
\begin{gathered}
\boldsymbol{\beta}\left(w_{k}^{-1}\right) \wedge \cdots \wedge \boldsymbol{\beta}\left(w_{1}^{-1}\right) \\
=\sum_{\boldsymbol{v} \in \mathcal{P}_{k}}[\boldsymbol{\beta}]_{\nu}^{k} \sum_{\tau \in S_{k}} \operatorname{sgn}(\tau) w_{k}^{-k+\tau(1)-v_{\tau(1)}} w_{k-1}^{-k+\tau(2)-v_{\tau(2)}} \cdots w_{1}^{-k+\tau(k)-v_{\tau(k)}}=\sum_{\nu \in \mathcal{P}_{k}}[\boldsymbol{\beta}]_{\nu}^{k} \Delta_{\nu}\left(\mathbf{w}_{k}^{-1}\right) \\
=\sum_{\nu \in \mathcal{P}_{k}}[\boldsymbol{\beta}]_{v}^{k} \cdot s_{\nu}\left(\mathbf{w}_{k}^{-1}\right) \Delta_{0}\left(\mathbf{w}_{k}^{-1}\right)=\sum_{\nu \in \mathcal{P}_{k}} s_{\nu}\left(\mathbf{w}_{k}^{-1}\right)[\boldsymbol{\beta}]_{v}^{k} \cdot \frac{\Delta_{0}\left(\mathbf{w}_{k}\right)}{w_{k}^{k-1} \cdots w_{1}^{k-1}},
\end{gathered}
$$

whence the claim, obtained by multiplying both (59) and (60) by $\left(\prod_{j=1}^{k} w_{j}^{k}\right) / \Delta_{0}\left(\mathbf{w}_{k}\right)$.

8.4 Lemma For all $u \in \bigwedge^{r} V\left[\left[\mathbf{w}_{k}, \mathbf{w}_{k}^{-1}\right]\right.$

$$
\sum_{\mu}\left([\mathbf{b}]_{\mu}^{k} \wedge u\right) s_{\boldsymbol{\mu}}\left(\mathbf{z}_{k}\right)=\bar{\sigma}_{+}\left(\mathbf{z}_{k}\right)[\mathbf{b}]_{0}^{k} \wedge u=\prod_{j=1}^{k} z_{j}^{r} \Gamma\left(\mathbf{z}_{k}\right) u .
$$

Proof We specified that the equality holds in $\bigwedge^{r} V\left[\left[\mathbf{w}_{k}, \mathbf{w}_{k}^{-1}\right]\right.$ to emphasize the supposed $\mathbb{Q}\left[\left[\mathbf{w}_{k}, \mathbf{w}_{k}^{-1}\right]\right.$ linearity of the Schubert derivation. This said, it is not restrictive to assume that $u$ is a basis element $[\mathbf{b}]_{\lambda}^{r}$ of $\bigwedge^{r} V$. The basic remark is that

$$
\begin{aligned}
\frac{1}{E_{k}\left(z_{1}\right)} \cdot \frac{1}{E_{k}\left(z_{2}\right)} \cdots \frac{1}{E_{k}\left(z_{k}\right)} & =\prod_{j=1}^{k}\left(1+h_{1} z_{j}+h_{z} z_{j}^{2}+h_{3} z_{j}^{3}+\cdots\right) \\
& =\sum_{\mu \in \mathcal{P}_{k}} s_{\mu}\left(\mathbf{z}_{k}\right) \Delta_{\lambda}\left(H_{k}\right),
\end{aligned}
$$

having used one of the declination of the celebrated Cauchy formula, as in [11, Proposition 2, (iii)], already employed in the proof of Lemma 8.2. Therefore:

$$
\begin{aligned}
\sum_{\mu}[\mathbf{b}]_{\mu}^{k} s_{\mu}\left(\mathbf{z}_{k}\right) & =\sum_{\mu}\left(\Delta_{\mu}\left(H_{k}\right) s_{\mu}\left(\mathbf{z}_{k}\right)\right)[\mathbf{b}]_{0}^{k} \\
& =\frac{1}{E_{k}\left(z_{1}\right) \cdots E_{k}\left(z_{k}\right)}[\mathbf{b}]_{0}^{k}=\sigma_{+}\left(\mathbf{z}_{k}\right)[\mathbf{b}]_{0}^{k},
\end{aligned}
$$

where in the last equality we have repeatedly used the module structure of $\bigwedge^{r} V$ over $B_{r}$. Then:

$$
\sum_{\mu}[\mathbf{b}]_{\mu}^{k} s_{\mu}\left(\mathbf{z}_{k}\right) \wedge u=\sigma_{+}\left(\mathbf{z}_{k}\right)[\mathbf{b}]_{0}^{k} \wedge u,
$$

and the result now follows from Theorem 6.5.

We can finally express the action of the generating function $\mathcal{E}_{\mu, v}\left(\mathbf{z}_{k}, \mathbf{w}_{k}^{-1}\right)$ on a basis element of $B_{r}$.

8.5 Theorem (First Version). For all $\lambda \in \mathcal{P}_{r}$ :

$$
\left(\mathcal{E}\left(\mathbf{z}_{k}, \mathbf{w}_{k}^{-1}\right) \Delta_{\lambda}\left(H_{r}\right)\right)[\mathbf{b}]_{0}^{r}=\prod_{j=1}^{k}\left(\frac{z_{j}}{w_{j}}\right)^{r-k} \Gamma\left(\mathbf{z}_{k}\right) \Gamma^{*}\left(\mathbf{w}_{k}\right)[\mathbf{b}]_{\lambda}^{r} .
$$


Proof We have

$$
\begin{aligned}
\mathcal{E}\left(\mathbf{z}_{k}, \mathbf{w}_{k}^{-1}\right)[\mathbf{b}]_{\lambda}^{r} & \left.=\sum_{\mu \in \mathcal{P}_{k}} s_{\boldsymbol{\mu}}\left(\mathbf{z}_{k}\right)[\mathbf{b}]_{\mu}^{k} \wedge \sum_{v \in \mathcal{P}_{k}} s_{\nu}\left(\mathbf{w}_{k}\right)\left([\boldsymbol{\beta}]_{v}^{k}\right\lrcorner[\mathbf{b}]_{\lambda}^{r}\right) & & \left(\text { definition of } \mathcal{E}\left(\mathbf{z}_{k}, \mathbf{w}_{k}^{-1}\right)\right) \\
& \left.=\sigma_{+}\left(\mathbf{z}_{k}\right)[\mathbf{b}]_{0}^{k} \wedge \frac{\prod_{j=1}^{k} w_{j}^{k-1}}{\Delta_{0}\left(\mathbf{w}_{k}\right)} \cdot \boldsymbol{\beta}\left(w_{k}^{-1}\right) \wedge \cdots \wedge \boldsymbol{\beta}\left(w_{1}^{-1}\right)\right\lrcorner[\mathbf{b}]_{\lambda}^{r} & & \text { (Lemmas 8.2 and 8.3) } \\
& =\sigma_{+}\left(\mathbf{z}_{k}\right)[\mathbf{b}]_{0}^{k} \wedge \frac{1}{\prod_{j=1}^{k} w_{j}^{r-k}} \Gamma^{*}\left(\mathbf{w}_{k}\right)[\mathbf{b}]_{\lambda}^{r} . & & \text { (Theorem 7.3) }
\end{aligned}
$$

Now we use the fact that $\Gamma^{*}\left(\mathbf{w}_{k}\right)[\mathbf{b}]_{\lambda}^{r}$ is a $\mathbb{Q}\left[\mathbf{w}_{k}, \mathbf{w}_{k}^{-1}\right]$-linear combination of $[\mathbf{b}]_{\mu}^{r-k}$ and then, by invoking Theorem 6.5, applied to last equality above, we obtain:

$$
\prod_{j=1}^{k}\left(\frac{z_{j}}{w_{j}}\right)^{r-k} \Gamma\left(\mathbf{z}_{k}\right) \Gamma^{*}\left(\mathbf{w}_{k}\right)[\mathbf{b}]_{\lambda}^{r},
$$

as announced.

8.6 Corollary If $r-k \geq \ell(\lambda)$ then

$$
\Gamma\left(\mathbf{z}_{k}\right) \Gamma^{*}\left(\mathbf{w}_{k}\right)[\mathbf{b}]_{\lambda}^{r}=\sigma_{+}\left(\mathbf{z}_{k}\right) \bar{\sigma}_{-}\left(\mathbf{z}_{k}\right) \bar{\sigma}_{+}\left(\mathbf{w}_{k}\right) \sigma_{-}\left(\mathbf{w}_{k}\right)[\mathbf{b}]_{\lambda}^{r} .
$$

Proof First of all notice that for every $\lambda \in \mathcal{P}_{r}$, it turns out that

$$
\bar{\sigma}_{+}\left(\mathbf{w}_{k}\right) \sigma_{-}\left(\mathbf{w}_{k}\right)[\mathbf{b}]_{\lambda}^{r-k}=\sum_{\boldsymbol{\mu} \in \mathcal{P}_{r}} a_{\boldsymbol{\mu}}\left(\mathbf{w}_{k}, \mathbf{w}_{k}^{-1}\right)[\mathbf{b}]_{\mu}^{r-k},
$$

where $a_{\boldsymbol{\mu}}\left(\mathbf{w}_{k}, \mathbf{w}_{k}^{-1}\right) \in \mathbb{Q}\left[\mathbf{w}_{k}, \mathbf{w}_{k}^{-1}\right]$. Then we have:

$$
\begin{aligned}
\Gamma\left(\mathbf{z}_{k}\right) \Gamma^{*}\left(\mathbf{w}_{k}\right)[\mathbf{b}]_{\lambda}^{r} & =\Gamma\left(\mathbf{z}_{k}\right) \bar{\sigma}_{+}\left(\mathbf{w}_{k}\right) \sigma_{-}\left(\mathbf{w}_{k}\right)[\mathbf{b}]_{\lambda}^{r-k} \\
& =\sum_{\mu \in \mathcal{P}_{r}} a_{\boldsymbol{\mu}}\left(\mathbf{w}_{k}, \mathbf{w}_{k}^{-1}\right) \Gamma\left(\mathbf{z}_{k}\right)[\mathbf{b}]_{\mu}^{r-k} \\
& =\sum_{\mu \in \mathcal{P}_{r}} a_{\mu}\left(\mathbf{w}_{k}, \mathbf{w}_{k}^{-1}\right)[\mathbf{b}]_{\lambda}^{r} \\
& =\sigma_{+}\left(\mathbf{z}_{k}\right) \bar{\sigma}_{-}\left(\mathbf{w}_{k}\right) \sum_{\mu \in \mathcal{P}_{r}} a_{\mu}\left(\mathbf{w}_{k}, \mathbf{w}_{k}^{-1}\right)[\mathbf{b}]_{\mu}^{r} \\
& =\sigma_{+}\left(\mathbf{z}_{k}\right) \bar{\sigma}_{-}\left(\mathbf{w}_{k}\right) \sigma_{+}\left(\mathbf{z}_{k}\right) \bar{\sigma}_{-}\left(\mathbf{w}_{k}\right)[\mathbf{b}]_{\lambda}^{r} .
\end{aligned}
$$

8.7 Remark If $\ell(\lambda)>r-k$, Corollary 8.6 fails. We have however the following uniform way to compute $\Gamma^{*}\left(\mathbf{w}_{k}\right)[\mathbf{b}]_{\lambda}^{r}$.

8.8 Proposition For all $\lambda \in \mathcal{P}_{r}$ and $k, r \geq 0$ :

$$
\Gamma^{*}\left(\mathbf{w}_{k}\right)[\mathbf{b}]_{\lambda}^{r}=\left|\begin{array}{cccc}
\frac{1}{w_{1}^{r-1+\lambda_{1}}} & \frac{1}{w_{1}^{r-2+\lambda_{2}}} & \cdots & \frac{1}{w_{1}^{\lambda_{r}}} \\
\vdots & \vdots & \ddots & \\
\frac{1}{w_{k}^{r-1+\lambda_{1}}} & \frac{1}{w_{k}^{r-2+\lambda_{2}}} & \cdots & \frac{1}{w_{k}^{\lambda_{r}}} \\
b_{r-1+\lambda_{1}} & b_{r-2+\lambda_{2}} & \cdots & b_{\lambda_{r}}
\end{array}\right| \in \bigwedge^{r-k} V
$$


using the same notation as in 4.1 .

Proof By Theorem 7.3

$$
\begin{aligned}
\Gamma^{*}\left(\mathbf{w}_{k}\right)[\mathbf{b}]_{\lambda}^{r} & \left.=\frac{\left(z_{1} \cdots z_{k}\right)^{r-1}}{\Delta_{0}\left(\mathbf{w}_{k}\right)}\left(\boldsymbol{\beta}\left(w_{k}^{-1}\right) \wedge \boldsymbol{\beta}\left(w_{k-1}^{-1}\right) \wedge \cdots \wedge \boldsymbol{\beta}\left(w_{1}^{-1}\right)\right)\right\lrcorner[\mathbf{b}]_{\lambda}^{r} \\
& =\frac{\left(z_{1} \cdots z_{k}\right)^{r-1}}{\Delta_{0}\left(\mathbf{w}_{k}\right)}\left|\begin{array}{cccc}
\frac{1}{w_{1}^{r-1+\lambda_{1}}} & \frac{1}{w_{1}^{r-2+\lambda_{2}}} & \cdots & \frac{1}{w_{1}^{\lambda_{r}}} \\
\vdots & \vdots & \ddots & 1 \\
\frac{1}{w_{k}^{r-1+\lambda_{1}}} & \frac{1}{w_{k}^{r-2+\lambda_{2}}} & \cdots & \frac{1}{w_{k}^{\lambda_{r}}} \\
b_{r-1+\lambda_{1}} & b_{r-2+\lambda_{2}} & \cdots & b_{\lambda_{r}}
\end{array}\right|
\end{aligned}
$$

\subsection{Another formulation}

Let us agree that

$$
w_{k}^{r-k} \cdots w_{2}^{r-2} w_{1}^{r-1} \Delta_{\lambda}\left(\mathbf{w}_{k}^{-1}, H_{r-k}\right)[\mathbf{b}]_{0}^{r}=\boldsymbol{\beta}\left(w_{k}^{-1}\right) \wedge \cdots \wedge \boldsymbol{\beta}\left(w_{1}^{-1}\right)_{\lrcorner}[\mathbf{b}]_{\lambda}^{r},
$$

defines $\Delta_{\lambda}\left(\mathbf{w}_{k}, H_{r-k}\right) \in B_{r}\left[w^{-1}\right]$. The expansion of (63) as a linear combinations of basis elements of $\bigwedge^{r-k} V$, Giambelli's formula (22) and the expansion rule of a determinant, easily imply that

$$
\Delta_{\lambda}\left(\mathbf{w}_{k}^{-1}, H_{r-k}\right)=\left|\begin{array}{cccc}
\frac{1}{w_{1}^{\lambda_{1}}} & \frac{1}{w_{1}^{\lambda_{2}-1}} & \cdots & \frac{1}{w_{1}^{\lambda_{r}-r+1}} \\
\vdots & \vdots & \ddots & \vdots \\
\frac{1}{w_{k}^{\lambda_{1}+k-1}} & \frac{1}{w_{k}^{\lambda_{2}+k-2}} & \cdots & \frac{1}{w_{k}^{\lambda_{r}+k-r}} \\
h_{\lambda_{1}+k} & h_{\lambda_{2}+k+1} & \cdots & h_{\lambda_{r}+k+r-1} \\
\vdots & \vdots & \ddots & \vdots \\
h_{\lambda_{1}+r-1} & h_{\lambda_{2}+r-2} & \cdots & h_{\lambda_{r}}
\end{array}\right|
$$

This enables to state a second version of 8.5 , which works well for practical purposes and generalises [20, Main Theorem 4.3].

8.10 Theorem (second version). The following equality holds: 


$$
\mathcal{E}\left(\mathbf{z}_{k}, \mathbf{w}_{k}^{-1}\right) \Delta_{\lambda}\left(H_{r}\right)=\prod_{j=1}^{k}\left(\frac{z_{j}}{w_{j}}\right)^{r-k} \frac{1}{E_{r}\left(z_{j}\right)} \Delta_{\lambda}\left(\mathbf{w}_{k}^{-1}, \bar{\sigma}_{-}(z) H_{r}\right)
$$

$$
=\prod_{j=1}^{k}\left(\frac{z_{j}}{w_{j}}\right)^{r-k} \frac{1}{E_{r}\left(z_{j}\right)}\left|\begin{array}{cccc}
\frac{1}{w_{1}^{\lambda_{1}}} & \frac{1}{w_{1}^{\lambda_{2}-1}} & \ldots & \frac{1}{w_{1}^{\lambda_{r}-r+1}} \\
\vdots & \vdots & \ddots & \\
\frac{1}{w_{k}^{\lambda_{1}+k-1}} & \frac{1}{w_{k}^{\lambda_{2}+k-2}} & \cdots & \frac{1}{w_{k}^{\lambda_{r}+k-r}} \\
\bar{\sigma}_{-}\left(\mathbf{z}_{k}\right) h_{\lambda_{1}+k} & \bar{\sigma}_{-}\left(\mathbf{z}_{k}\right) h_{\lambda_{2}+k+1} & \ldots & \bar{\sigma}_{-}\left(\mathbf{z}_{k}\right) h_{\lambda_{r}+k+r-1} \\
\vdots & \vdots & \ddots & \vdots \\
\bar{\sigma}_{-}\left(\mathbf{z}_{k}\right) h_{\lambda_{1}+r-1} & \bar{\sigma}_{-}\left(\mathbf{z}_{k}\right) h_{\lambda_{2}+r-2} & \ldots & \bar{\sigma}_{-}\left(\mathbf{z}_{k}\right) h_{\lambda_{r}}
\end{array}\right|,
$$

where

$$
\bar{\sigma}_{-}\left(\mathbf{z}_{k}\right) h_{j}=h_{j}-e_{1}\left(\mathbf{z}_{k}^{-1}\right) h_{j-1}+\cdots+(-1)^{k} e_{k}\left(\mathbf{z}_{k}^{-1}\right) h_{j-k} .
$$

Proof By Theorem 8.5 we have:

$$
\begin{aligned}
& \mathcal{E}\left(\mathbf{z}_{k}, \mathbf{w}_{k}^{-1}\right) \Delta_{\lambda}\left(H_{r}\right)=\prod_{j=1}^{k}\left(\frac{z_{j}}{w_{j}}\right)^{r-k} \Gamma\left(\mathbf{z}_{k}\right) \Gamma^{*}\left(\mathbf{w}_{k}\right) \Delta_{\lambda}\left(H_{r}\right) \quad \text { (Theorem 8.5) } \\
& =\prod_{j=1}^{k}\left(\frac{z_{j}}{w_{j}}\right)^{r-k} \Gamma\left(\mathbf{z}_{k}\right) \Delta_{\lambda}\left(\mathbf{w}_{k}, H_{r-k}\right) \quad \text { (Proposition } 8.8 \text { andequation (64)) } \\
& \left.=\prod_{j=1}^{k}\left(\frac{z_{j}}{w_{j}}\right)^{r-k} \sigma_{+}\left(\mathbf{z}_{k}\right) \bar{\sigma}_{-}\left(\mathbf{z}_{k}\right) \Delta_{\lambda}\left(\mathbf{w}_{k}, H_{r}\right) \quad \text { (Def inition } 4.3-(30) \text { of } \Gamma\left(\mathbf{z}_{k}\right)\right) \\
& =\prod_{j=1}^{k}\left(\frac{z_{j}}{w_{j}}\right)^{r-k} \frac{1}{E_{r}\left(z_{j}\right)} \cdot \Delta_{\lambda}\left(\mathbf{w}_{k}, \bar{\sigma}_{-}\left(\mathbf{z}_{k}\right) H_{r}\right) \quad \text { (Definition } 3.4 \text { of the } \mathrm{B}_{\mathrm{r}}-\text { module structure of } \bigwedge^{r} V \text { and Proposition 3.6) }
\end{aligned}
$$

as desired. Expression (66) for $\bar{\sigma}_{-}\left(\mathbf{z}_{k}\right) h_{j}$ is Lemma 6.2.

8.11 Remark It is a good point to remark here that for $k=1$, Theorem 8.10 above is a special case of a more general result due to the first author and Nasrollah Nejad [3], showing that the universal factorisation algebra of a generic polynomial itself carries a structure over the Lie algebra of endomorphism of a suitable module keeping track of the indeterminate coefficients of the polynomial.

\subsection{Exponential form}

Finally, let us define, as it is customary, new formal variable $\left(x_{j}\right)_{j \geq 1}$ through the equality:

$$
\exp \left(\sum_{j \geq 0} x_{j} z^{j}\right)=\frac{1}{E_{r}(z)} .
$$

In this case one can write

$$
\prod_{j=1}^{k} \frac{1}{E_{r}\left(z_{j}\right)}=\exp \left(\sum_{j=0} x_{j} p_{j}\left(\mathbf{z}_{k}\right)\right)
$$


where $p_{j}\left(\mathbf{z}_{k}\right)=z_{1}^{j}+\cdots+z_{k}^{j}$ is the $i$-th power sum symmetric polynomial in $\left(z_{1}, \ldots, z_{k}\right)$ and where $x_{i}$ is precisely the $i$-th degree power sum in the $r$ universal roots $\left(y_{1}, \ldots, y_{r}\right)$ of the polynomial $E_{r}(z)$, i.e. $E_{r}(z)=\prod_{i=1}^{r}\left(1-y_{i} z\right)$ in the universal splitting $\mathbb{Q}$-algebra for the polynomial $E_{r}(z) \in \mathbb{Q}[z]$. This allows to shape our result in the form

\subsection{Corollary We have:}

$$
\mathcal{E}\left(\mathbf{z}_{k}, \mathbf{w}_{k}^{-1}\right) \Delta_{\lambda}\left(H_{r}\right)=\prod_{j=1}^{k}\left(\frac{z_{j}}{w_{j}}\right)^{r-k} \exp \left(\sum_{j=0} x_{j} p_{j}\left(\mathbf{z}_{k}\right)\right) \Delta_{\lambda}\left(\mathbf{w}_{k}, \bar{\sigma}_{-}\left(\mathbf{z}_{k}\right) H_{r-k}\right) .
$$

Formula (67) is easy to use for practical computations of the $g l\left(\bigwedge^{k} V\right)$-representation of $B_{r}$, for those special case of $k$ and $r$ that everybody may possibly need.

Acknowledgements This work is an expansion of part of the Ph.D. thesis of the first author performed during her hosting at the Department of Mathematical Sciences of Politecnico of Torino under the sponsorship of Ministry of Science of the Islamic Republic of Iran and of Finanziamento Diffuso della Ricerca, No. 53_RBA17GATLET. The second and fourth authors stay enjoyed the full sponsorship of the above Finanziamento Diffuso (No. 53_RBA17GATLET) and Progetto di Eccellenza del Dipartimento di Scienze Matematiche, 2018-2022, No. E11G18000350001. The project also benefitted the partial support of INDAM-GNSAGA, PRIN "Geometria delle Varietà Algebriche". We owe a lot to the precious referee's comment which helped us to improve the presentation of our paper. For discussions and criticisms, however, we want to primarily thank Inna Scherbak, who first suggested us to generalise the DJKM picture in the way as now stands in the present paper. Finally, we are indebted to Joachim Kock and Andrea T. Ricolfi for carefully reading and many other kinds of assistance.

Funding Open access funding provided by Politecnico di Torino within the CRUI-CARE Agreement.

Open Access This article is licensed under a Creative Commons Attribution 4.0 International License, which permits use, sharing, adaptation, distribution and reproduction in any medium or format, as long as you give appropriate credit to the original author(s) and the source, provide a link to the Creative Commons licence, and indicate if changes were made. The images or other third party material in this article are included in the article's Creative Commons licence, unless indicated otherwise in a credit line to the material. If material is not included in the article's Creative Commons licence and your intended use is not permitted by statutory regulation or exceeds the permitted use, you will need to obtain permission directly from the copyright holder. To view a copy of this licence, visit http://creativecommons.org/licenses/by/4.0/.

\section{References}

1. Anderson, D., Nigro, A.: Minuscule Schubert Calculus and the geometric Satake correspondence. In: Hu J., Li C., Mihalcea L.C. (Eds.) Schubert Calculus and Its Applications in Combinatorics and Representation Theory, ICTSC 2017. Springer Proceedings in Mathematics and Statistics, vol 332. Springer, Singapore. https://doi.org/10.1007/978-981-15-7451-1_6, ArXiv:1907.08102.pdf

2. Behzad, O., Gatto, L.: Bosonic and Fermionic representations of endomorphisms of exterior Algebras. Fundamenta Math. (2021) (to appear). ArXiv:2009.00479.pdf

3. Behzad, O., Nasrollah, A.: Universal Factorisation algebras of polynomials represent Lie algebras of endomorphisms. J. Algebra Appl. (2021). https://doi.org/10.1142/S0219498822500724, arXiv :2006.07893

4. Cordovez, J., Gatto, L., Santiago, T.: Newton binomial formulas in Schubert calculus. Rev. Mat. Complut. 22(1), 129-152 (2009)

5. Costa, L., Marchesi, S., Miró-Roig, R.M.: Tango bundles on Grassmannians. Math. Nachr. 289(8-9), 950-961 (2016)

6. Cotti, G., Dubrovin, B., Guzzetti, D.: Helix structures in quantum cohomology of fano varieties ArXiv :1811.09235 (2019) 
7. Cox, B., Futorny, V., Tirao, J.A.: DJKM algebras and non-classical orthogonal polynomials. J. Differ. Equ. 255(9), 2846-2870 (2013)

8. Date, E., Jimbo, M., Kashiwara, M., Miwa, T.: Transformation groups for soliton equations. III. Operator approach to the Kadomtsev-Petviashvili equation. J. Phys. Soc. Jpn 50(11), 3806-3812 (1981)

9. Frenkel, E., Ben-Zvi, D.: Vertex Algebras and Algebraic Curves, Mathematical Surveys and Monographs, vol. 88, 2nd edn. American Mathematical Society, Providence, RI (2004)

10. Frenkel, I., Penkov, I., Serganova, V.: A categorification of the boson-fermion correspondence via representation theory of $s l(\infty)$. Commun. Math. Phys. 341(3), 911-931 (2016)

11. Fulton, W.: Young tableaux, London Mathematical Society Student Texts, vol. 35. Cambridge University Press, Cambridge (with applications to representation theory and geometry) (1997)

12. Macdonald, I.G.: Symmetric Functions and Hall Polynomials, Oxford Classic Texts in the Physical Sciences, 2nd edn. The Clarendon Press, Oxford University Press, New York, With contribution by A.V. Zelevinsky and a foreword by Richard Stanley, Reprint of the 2008 paperback edition (2015)

13. Gatto, L.: Schubert calculus via Hasse-Schmidt derivations. Asian J. Math. 9(3), 315-321 (2005)

14. Gatto, L., Laksov, D.: From linear recurrence relations to linear ODEs with constant coefficients. J. Algebra Its Appl. 15(6), 1650109 (2016). https://doi.org/10.1142/S0219498816501097. (23 pages)

15. Gatto, L., Ricolfi, A.T.: Jet bundles on Gornstein curves and applications. J. Singul. 21, 70-103 (2020)

16. Gatto, L., Rowen, L.: Grassman semialgebras and the Cayley-Hamilton theorem. Proc. AMS 7, 183201 (2020). https://doi.org/10.1090/bproc/53. ArXiv:1803.08093

17. Gatto, L., Salehyan, P.: The boson-fermion correspondence from linear ODEs. J. Algebra 415, 162183 (2014)

18. Gatto, L., Salehyan, P.: Hasse-Schmidt derivations on Grassmann Algebras, IMPA Monographs, vol. 4. Springer, Cham (with applications to vertex operators) (2016)

19. Gatto, L., Salehyan, P.: On Plücker equations characterizing Grassmann cones, Schubert varieties, equivariant cohomology and characteristic classes-IMPANGA 15, EMS Ser. Congr. Rep., Eur. Math. Soc., Zürich, pp. 97-125, arXiv:1603.00510 (2018)

20. Gatto, L., Salehyan, P.: The cohomology of the Grassmannian is a $g l_{n}$-module. Commun. Algebra 48(1), 274-290 (2020)

21. Gatto, L., Salehyan, P.: Schubert derivations on the infinite exterior power. Bull. Braz. Math. Soc. New Ser. (2020). https://doi.org/10.1007/s00574-020-00195-9

22. Gatto, L., Santiago, T.: Equivariant Schubert calculus. Ark. Mat. 48(1), 41-55 (2010)

23. Gatto, L., Scherbak, I.: Hasse-Schmidt derivations and Cayley-Hamilton theorem for exterior algebras. Functional analysis and geometry: Selim Grigorievich Krein centennial, Contemp. Math., vol. 733, American Mathematical Society, Providence, RI, 2019, pp. 149-165

24. Jimbo, M., Miwa, T.: Solitons and infinite-dimensional Lie algebras. Publ. Res. Inst. Math. Sci. 19(3), 943-1001 (1983)

25. Kac, V.G., Raina, A.K., Rozhkovskaya, N.: Bombay Lectures on Highest Weight Representations of Infinite Dimensional Lie Algebras, Advanced Series in Mathematical Physics, vol. 29, 2nd edn. World Scientific Publishing Co. Pvt. Ltd., Hackensack (2013)

26. Laksov, D.: Schubert calculus and equivariant cohomology of Grassmannians. Adv. Math. 217(4), 1869-1888 (2008)

27. Laksov, D., Thorup, A.: A determinantal formula for the exterior powers of the polynomial ring. Indiana Univ. Math. J. 56(2), 825-845 (2007)

28. Tian, Y.: Towards a categorical boson-fermion correspondence. Adv. Math. 365, 107034 (2020)

Publisher's Note Springer Nature remains neutral with regard to jurisdictional claims in published maps and institutional affiliations. 